\title{
A CALDERÓN MULTIPLICATIVE PRECONDITIONER FOR COUPLED SURFACE- VOLUME ELECTRIC FIELD INTEGRAL EQUATIONS
}

Hakan Bağc1 ${ }^{(1)}$, Francesco P. Andriulli ${ }^{(2)}$, Kristof Cools $^{(3)}$, Femke Olyslager ${ }^{(3)}$, and Eric Michielssen $^{(1)}$

(1) Radiation Laboratory, Department of Electrical Engineering and Computer Science, University of Michigan, Ann Arbor, MI

(2) Antenna and EMC Lab, Electronics Department, Politecnico di Torino, Torino, Italy

(3) Electromagnetics Group, Department of Information Technology, Ghent University, Ghent, Belgium

Email: $\underline{\text { bagci@umich.edu }}$

\begin{abstract}
A well-conditioned coupled set of surface (S) and volume (V) electric field integral equations (SEFIE and V-EFIE) for analyzing wave interactions with densely discretized composite structures is presented. Whereas the V-EFIE operator is well-posed even when applied to densely discretized volumes, a classically formulated S-EFIE operator is ill-posed when applied to densely discretized surfaces. This renders the discretized coupled S-EFIE and V-EFIE system illconditioned, and its iterative solution inefficient or even impossible. The proposed scheme regularizes the coupled set of S-EFIE and V-EFIE using a Calderón multiplicative preconditioner (CMP)-based technique. The resulting scheme enables the efficient analysis of electromagnetic interactions with composite structures containing fine/subwavelength geometric features. Numerical examples demonstrate the efficiency of the proposed scheme.
\end{abstract}

Keywords: surface electric field integral equation, volume electric field integral equations, multiplicative preconditioning, Calderón preconditioning 


\section{Introduction}

Integral equation techniques for modeling electromagnetic interactions with composite structures comprised of perfect electrically conducting (PEC) surfaces and dielectric volumes have many practical applications. These techniques often seek the solution of a coupled set of surface (S) and volume (V) electric field integral equations (EFIEs) that enforce electric field boundary and consistency conditions on PEC surfaces and throughout dielectric volumes, respectively [1-3]. To permit the analysis of structures with sub-wavelength geometric features, e.g. microwave circuits and complex antenna feeds, these techniques should apply robustly to PEC surfaces and dielectric volumes approximated by locally or globally-dense spatial meshes.

While V-EFIE operators are bounded and well-posed even when applied to densely discretized volumes $[4,5]$, S-EFIE operators become ill-posed when applied to densely discretized surfaces [6-10]. As a result, the methods-of-moments (MOM) systems obtained upon discretizing coupled sets of S- and V-EFIEs applied to structures involving densely discretized PEC surfaces tend to be ill-conditioned and their iterative solution becomes prohibitively expensive.

In recent years, many techniques that leverage Calderón identities to alleviate the ill-posedness of S-EFIE operators have been proposed [6-10]. These techniques exploit the self-regularizing property of the S-EFIE, i.e. the fact that its square has a bounded spectrum, thus giving rise to MOM matrices that are well-conditioned, independent of the surface discretization density. Unfortunately, many of these methods suffer from implementation difficulties related to the fact that the EFIE operators' product needs to be discretized [8]. Various methods that use ad hoc integration rules and/or operational manipulations have been used for this purpose $[7,9,10]$; unfortunately none of them is easily integrated into existing MOM codes that discretize the SEFIE using the well-known Rao-Wilton-Glisson (RWG) functions [11].

This paper presents a well-conditioned coupled set of S- and V-EFIEs. The first equation of the coupled set imposes electric field boundary conditions on PEC surfaces and is regularized by the S-EFIE operator. The resulting equation calls for the discretization of S-EFIE/S-EFIE and SEFIE/V-EFIE operator products, which is accomplished using a Calderón multiplicative preconditioner (CMP)-based technique [8]. The second equation of the coupled set links electric fields and polarization currents throughout dielectric volumes. The proposed approach preserves the original CMP's multiplicative nature and requires only a standard RWG and SchaubertWilton-Glisson (SWG) based discretization [12] of the surfaces and volumes. As a result, the 
proposed preconditioner is easily implemented into existing MOM codes and the resulting solver can trivially be accelerated via available fast matrix-vector multiplication methods including the adaptive integral method (AIM) [13], multilevel fast multipole algorithm (MLFMA) [14, 15], and their parallelized versions.

This paper is organized as follows. Section II formulates a coupled set of S- and V-EFIEs and details its CMP-based discretization. Section III verifies the effectiveness of the proposed regularization technique by applying it to structures with sub-wavelength features, viz., a dielectric-filled waveguide slot antenna on a shuttle model and dielectric antennas with finefeatured metallic feeds. Section IV presents conclusions and avenues for future research.

\section{Formulation}

This section describes the proposed CMP regularization technique for the coupled set of S- and V-EFIEs. Section II-A formulates the set of S-EFIE and V-EFIE in surface current and electric flux densities and details its standard MOM-based discretization. Section II-B describes the proposed CMP regularizer.

\section{A. Coupled Set of S- and V-EFIEs and its MOM Discretization}

Consider a composite structure comprising PEC surfaces $S$ and potentially inhomogeneous dielectric volumes $V$ that reside in free space (Fig. 1). Let $\varepsilon(\mathbf{r})$ and $\varepsilon_{0}$ denote the permittivity of $V$ and free-space, respectively; let $\mu_{0}$ denote the permeability of all of space. A timeharmonic electric field $\mathbf{E}^{\mathrm{inc}}(\mathbf{r})$ excites $S$ and $V$; here and in what follows, time dependence $e^{-i \omega t}$ is assumed and suppressed. The surface and volume (polarization) current densities $\mathbf{J}^{\mathrm{s}}(\mathbf{r})$ and $\mathbf{J}^{\mathrm{v}}(\mathbf{r})$ induced on $S$ and in $V$ generate the scattered electric field $\mathbf{E}^{\text {sca }}(\mathbf{r})$. Enforcing electric field boundary and consistency conditions on $S$ and in $V$ yields

$$
\begin{gathered}
\hat{\mathbf{n}}(\mathbf{r}) \times \mathbf{E}^{\text {sca }}(\mathbf{r})=\hat{\mathbf{n}}(\mathbf{r}) \times\left[L^{\mathrm{s}} \mathbf{J}^{\mathrm{s}}+L^{\mathrm{v}, \mathrm{J}} \mathbf{J}^{\mathrm{v}}\right]=-\hat{\mathbf{n}}(\mathbf{r}) \times \mathbf{E}^{\mathrm{inc}}(\mathbf{r}) \quad \forall \mathbf{r} \in S, \\
\mathbf{E}^{\mathrm{sca}}(\mathbf{r})-\mathbf{E}(\mathbf{r})=L^{\mathrm{s}} \mathbf{J}^{\mathrm{s}}+L^{\mathrm{v}, \mathrm{J}} \mathbf{J}^{\mathrm{v}}-\mathbf{E}(\mathbf{r})=-\mathbf{E}^{\mathrm{inc}}(\mathbf{r}) \quad \forall \mathbf{r} \in V .
\end{gathered}
$$

Here, $\mathbf{E}(\mathbf{r})=\mathbf{E}^{\text {inc }}(\mathbf{r})+\mathbf{E}^{\text {sca }}(\mathbf{r})$ denotes the total electric field, $\hat{\mathbf{n}}(\mathbf{r})$ is a unit normal to $S$, and the operators $L^{\mathrm{s}}$ and $L^{\mathrm{v}}$ are

$$
L^{\mathrm{s}} \mathbf{J}^{\mathrm{s}}=i \omega \mu_{0} \int_{S} G\left(\mathbf{r}, \mathbf{r}^{\prime}\right) \mathbf{J}^{\mathrm{s}}\left(\mathbf{r}^{\prime}\right) d \mathbf{r}^{\prime}-\frac{1}{i \omega \varepsilon_{0}} \nabla \int_{S} G\left(\mathbf{r}, \mathbf{r}^{\prime}\right) \nabla_{s}^{\prime} \cdot \mathbf{J}^{\mathrm{s}}\left(\mathbf{r}^{\prime}\right) d \mathbf{r}^{\prime},
$$

and 


$$
L^{\mathrm{v}, \mathrm{J}} \mathbf{J}^{\mathrm{v}}=i \omega \mu_{0} \int_{V} G\left(\mathbf{r}, \mathbf{r}^{\prime}\right) \mathbf{J}^{\mathrm{v}}\left(\mathbf{r}^{\prime}\right) d \mathbf{r}^{\prime}-\frac{1}{i \omega \varepsilon_{0}} \nabla \int_{V} G\left(\mathbf{r}, \mathbf{r}^{\prime}\right) \nabla^{\prime} \cdot \mathbf{J}^{\mathrm{v}}\left(\mathbf{r}^{\prime}\right) d \mathbf{r}^{\prime}
$$

In (3) and (4), $G\left(\mathbf{r}, \mathbf{r}^{\prime}\right)=e^{i k\left|\mathbf{r}-\mathbf{r}^{\prime}\right|} /\left(4 \pi\left|\mathbf{r}-\mathbf{r}^{\prime}\right|\right)$ is the free-space Green function. In $V, \mathbf{J}^{\mathbf{v}}(\mathbf{r})$ and $\mathbf{E}(\mathbf{r})$ are related as

$$
\mathbf{J}^{\mathrm{v}}(\mathbf{r})=-i \omega \chi(\mathbf{r}) \mathbf{D}(\mathbf{r})=-i \omega \chi(\mathbf{r}) \varepsilon(\mathbf{r}) \mathbf{E}(\mathbf{r}, t) \quad \forall \mathbf{r} \in V,
$$

where $\chi(\mathbf{r})=1-\varepsilon_{0} / \varepsilon(\mathbf{r})$ is the contrast parameter [12] and $\mathbf{D}(\mathbf{r})=\varepsilon(\mathbf{r}) \mathbf{E}(\mathbf{r})$ is the electric flux density. Inserting (5) into (1) and (2) yields the coupled set of S- and V-EFIEs in $\mathbf{J}^{\mathbf{s}}(\mathbf{r})$ and $\mathbf{D}(\mathbf{r})$ :

$$
\begin{gathered}
\hat{\mathbf{n}}(\mathbf{r}) \times\left[L^{\mathrm{s}} \mathbf{J}^{\mathrm{s}}+L^{\mathrm{v}, \mathrm{D}} \mathbf{D}\right]=-\hat{\mathbf{n}}(\mathbf{r}) \times \mathbf{E}^{\mathrm{inc}}(\mathbf{r}) \quad \forall \mathbf{r} \in S, \\
L^{\mathrm{s}} \mathbf{J}^{\mathrm{s}}+L^{\mathrm{v}, \mathrm{I}} \mathbf{D}=-\mathbf{E}^{\mathrm{inc}}(\mathbf{r}) \quad \forall \mathbf{r} \in V .
\end{gathered}
$$

Here the operators $L^{\mathrm{v}, \mathrm{D}}$ and $L^{\mathrm{v}, \mathrm{I}}$, which complement the operator $L^{\mathrm{v}, \mathrm{J}}$, are

$$
\begin{gathered}
L^{\mathrm{v}, \mathrm{D}} \mathbf{D}=i \omega \mu_{0} \int_{V} G\left(\mathbf{r}, \mathbf{r}^{\prime}\right)\left[-i \omega \chi\left(\mathbf{r}^{\prime}\right) \mathbf{D}\left(\mathbf{r}^{\prime}\right)\right] d \mathbf{r}^{\prime}-\frac{1}{i \omega \varepsilon_{0}} \nabla \int_{V} G\left(\mathbf{r}, \mathbf{r}^{\prime}\right) \nabla^{\prime} \cdot\left[-i \omega \chi\left(\mathbf{r}^{\prime}\right) \mathbf{D}\left(\mathbf{r}^{\prime}\right)\right] d \mathbf{r}^{\prime}, \\
L^{\mathrm{v}, \mathrm{I}} \mathbf{D}=L^{\mathrm{v}, \mathrm{D}} \mathbf{D}-\mathbf{D}(\mathbf{r}) / \varepsilon(\mathbf{r}) .
\end{gathered}
$$

To numerically solve (6) and (7), $S$ and $V$ are approximated by meshes of planar triangles (with smallest edge size $\delta^{\mathrm{s}}$ ) and tetrahedrons (with smallest edge size $\left.\delta^{\mathrm{v}}\right)$, and $\mathbf{J}^{\mathrm{s}}(\mathbf{r})$ and $\mathbf{D}(\mathbf{r})$ are approximated as

$$
\begin{gathered}
\mathbf{J}^{\mathrm{s}}(\mathbf{r}) \cong \sum_{k^{\prime}=1}^{N^{\mathrm{RWG}}} I_{k^{\prime}}^{\mathrm{RWG}} \mathbf{f}_{k^{\prime}}^{\mathrm{RWG}}(\mathbf{r}), \\
\mathbf{D}(\mathbf{r}) \cong \sum_{k^{\prime}=1}^{N^{\mathrm{WWG}}} I_{k^{\prime}}^{\mathrm{SWG}} \mathbf{f}_{k^{\prime}}^{\mathrm{SWG}}(\mathbf{r}),
\end{gathered}
$$

where $I_{k}^{\mathrm{RWG}}, k=1, \ldots, N^{\mathrm{RWG}}$ and $I_{k}^{\mathrm{SWG}}, k=1, \ldots, N^{\mathrm{SWG}}$ are unknown expansion coefficients, $\mathbf{f}_{k}^{\mathrm{RWG}}(\mathbf{r}), k=1, \ldots, N^{\mathrm{RWG}}$ are zeroth-order div-conforming RWG surface basis functions [Fig. 2(a)] [11] defined on pairs of triangles, and $\mathbf{f}_{k}^{\mathrm{SWG}}(\mathbf{r}), k=1, \ldots, N^{\mathrm{SWG}}$ are zeroth-order divconforming SWG volume basis functions [12] defined on tetrahedron facets. To determine the coefficients $I_{k}^{\mathrm{RWG}}$ and $I_{k}^{\mathrm{SWG}}$, (10) and (11) are inserted into (6) and (7), and the resulting equations are tested by curl-conforming $\hat{\mathbf{n}}(\mathbf{r}) \times \mathbf{f}_{k}^{\mathrm{RWG}}(\mathbf{r}), \quad k=1, \ldots, N^{\mathrm{RWG}}[$ Fig. 2(b)] and $\chi(\mathbf{r}) \mathbf{f}_{k}^{\mathrm{SWG}}(\mathbf{r}), \quad k=1, \ldots, N^{\mathrm{SWG}}$; this produces the linear system of equations of dimension $N^{\mathrm{RWG}}+N^{\mathrm{SWG}}$

$$
\mathbf{Z I}=\mathbf{V} .
$$


Here $\mathbf{I}$ and $\mathbf{V}$ are vectors of expansion coefficients and tested incident fields, respectively; their entries are

$$
\begin{aligned}
& \mathbf{I}_{k}=\left\{\begin{array}{ll}
I_{k}^{\mathrm{RWG}}, & k \leq N^{\mathrm{RWG}} \\
I_{k-N^{\mathrm{RWG}}}^{\mathrm{SWG}}, & \text { else }
\end{array},\right. \\
& \mathbf{V}_{k}=\left\{\begin{array}{ll}
-\left\langle\hat{\mathbf{n}}(\mathbf{r}) \times \mathbf{f}_{k}^{\mathrm{RWG}}(\mathbf{r}), \hat{\mathbf{n}}(\mathbf{r}) \times \mathbf{E}^{\mathrm{inc}}(\mathbf{r})\right\rangle_{\mathrm{s}}, & k \leq N^{\mathrm{RWG}}, \\
-\left\langle\chi(\mathbf{r}) \mathbf{f}_{k-N^{\mathrm{RWG}}}^{\mathrm{SWG}}(\mathbf{r}), \mathbf{E}^{\mathrm{inc}}(\mathbf{r})\right\rangle_{\mathrm{v}}, & \text { else }
\end{array},\right.
\end{aligned}
$$

with $\langle\mathbf{a}(\mathbf{r}), \mathbf{b}(\mathbf{r})\rangle_{\mathrm{s} / \mathrm{v}}=\int_{S / V} \mathbf{a}(\mathbf{r}) \cdot \mathbf{b}(\mathbf{r}) d \mathbf{r}$. The impedance matrix $\mathbf{Z}$ in (12) can be decomposed as

$$
\mathbf{Z}=\left[\begin{array}{ll}
\mathbf{Z}^{\text {RWG/RWG }} & \mathbf{Z}^{\text {RWG/SWG }} \\
\mathbf{Z}^{\text {SWG/RWG }} & \mathbf{Z}^{\text {SWG/SWG }}
\end{array}\right],
$$

where $\mathbf{Z}^{\mathrm{RWG} / \mathrm{RWG}}, \mathbf{Z}^{\mathrm{SWG} / \mathrm{SWG}}, \mathbf{Z}^{\mathrm{RWG} / \mathrm{SWG}}$, and $\mathbf{Z}^{\mathrm{SWG} / \mathrm{RWG}}$ account for surface test-surface basis, volume test-volume basis, surface test-volume basis, and volume test-surface basis interactions, respectively. Their entries are

$$
\begin{gathered}
\mathbf{Z}_{k, k^{\prime}}^{\mathrm{RWG} / R W G}=\left\langle\hat{\mathbf{n}}(\mathbf{r}) \times \mathbf{f}_{k}^{\mathrm{RWG}}(\mathbf{r}), \hat{\mathbf{n}}(\mathbf{r}) \times L^{\mathrm{s}} \mathbf{f}_{k^{\prime}}^{\mathrm{RWG}}\right\rangle_{\mathrm{s}}, \\
\mathbf{Z}_{k, k^{\prime}}^{\mathrm{SWGGG}}=\left\langle\chi(\mathbf{r}) \mathbf{f}_{k}^{\mathrm{SWG}}(\mathbf{r}), \hat{\mathbf{n}}(\mathbf{r}) \times L^{\mathrm{s}} \mathbf{f}_{k^{\prime}}^{\mathrm{RWG}}\right\rangle_{\mathrm{v}}, \\
\mathbf{Z}_{k, k^{\prime}}^{\mathrm{RWWG} / \mathrm{SWG}}=\left\langle\hat{\mathbf{n}}(\mathbf{r}) \times \mathbf{f}_{k}^{\mathrm{RWG}}(\mathbf{r}), L^{\left.\mathrm{v}, \mathrm{D} \mathbf{f}_{k^{\prime}}^{\mathrm{SWG}}\right\rangle_{\mathrm{s}},}\right. \\
\mathbf{Z}_{k, k^{\prime}}^{\mathrm{SWG} / \mathrm{SWG}}=\left\langle\chi(\mathbf{r}) \mathbf{f}_{k}^{\mathrm{SWG}}(\mathbf{r}), L^{\mathrm{v}, \mathrm{I}} \mathbf{f}_{k^{\prime}}^{\mathrm{SWG}}\right\rangle_{\mathrm{v}} .
\end{gathered}
$$

When analyzing electrically large and/or complex structures, i.e., when $N^{\mathrm{RWG}}+N^{\mathrm{SWG}}$ is large, (12) cannot be solved directly and iterative solves are called for. The computational cost of solving (12) iteratively scales multiplicatively with the cost of applying the impedance matrix $\mathbf{Z}$ to a trial solution vector and the number of iterations required to reach a specified residual. The cost of a matrix-vector multiplication always can be reduced by using adaptive integral [13] or multilevel fast multipole $[14,15]$ accelerators. The required number of iterations typically scales with $\mathbf{Z}$ 's condition number with small condition numbers guaranteeing fast convergence of the iterative solver.

The conditioning of $\mathbf{Z}$ depends on the spectral properties of the S- and V-EFIE operators $\hat{\mathbf{n}}(\mathbf{r}) \times L^{\mathrm{s}}$ and $L^{\mathrm{v}, \mathrm{I}}$. The spectral properties of the S-EFIE operator are well-documented: it is known that its singular values accumulate at zero and infinity as it contains a singular operator (the first integral in (3), i.e., the vector potential contribution) and a hypersingular operator (the second integral in (3), i.e. the scalar potential contribution) [6]; in other words, the S-EFIE 
operator is unbounded. As a result, $\mathbf{Z}^{\mathrm{RWG} / \mathrm{RWG}}$ is increasingly ill-conditioned when $\delta^{\mathrm{s}} \rightarrow 0$. Unlike the S-EFIE operator, the V-EFIE operator's spectrum is bounded [4,5]. The scalar potential contribution of the V-EFIE operator is Cauchy-singular (but not hypersingular) and its dominant contribution results from a volume integral. It has been shown in $[4,5]$ that matrices resulting from the discretization of V-EFIE operator are well-conditioned regardless of the discretization density. This means that $\mathbf{Z}^{\mathrm{SWG} / \mathrm{SWG}}$ is well-conditioned even when $\delta^{\mathrm{v}} \rightarrow 0$. Unfortunately, $\mathbf{Z}^{\text {RWG/RWG }}$ alone renders $\mathbf{Z}$ ill-conditioned and the iterative solution of (12) prohibitively expensive or even impossible in the presence of dense discretizations.

\section{B. Calderon Regularization and CMP-based Discretization of Hybrid Set of S- and V-EFIEs}

The unbounded nature of the S-EFIE operator can be cured using the well-known Calderón identity [16]

$$
\hat{\mathbf{n}}(\mathbf{r}) \times L^{\mathrm{s}}\left(\hat{\mathbf{n}}(\mathbf{r}) \times L^{\mathrm{s}}\right)=-\frac{1}{4}+K K=\left(K-\frac{1}{2}\right)\left(K+\frac{1}{2}\right) .
$$

Here $K \mathbf{J}^{\mathrm{s}}=\hat{\mathbf{n}}(\mathbf{r}) \times \nabla \times \int G\left(\mathbf{r}, \mathbf{r}^{\prime}\right) \mathbf{J}^{\mathrm{s}}\left(\mathbf{r}^{\prime}\right) d \mathbf{r}^{\prime}$ is a compact operator when acting on smooth surfaces [6]; this makes $\hat{\mathbf{n}}(\mathbf{r}) \times{ }^{\mathrm{s}} L^{\mathrm{s}}\left(\hat{\mathbf{n}}(\mathbf{r}) \times L^{\mathrm{s}}\right)$ a second kind operator. Therefore, (6) can be regularized using $\hat{\mathbf{n}}(\mathbf{r}) \times L^{\mathrm{s}}$ and the set of equations

$$
\begin{gathered}
\hat{\mathbf{n}}(\mathbf{r}) \times L^{\mathrm{s}}\left(\hat{\mathbf{n}}(\mathbf{r}) \times L^{\mathrm{s}}\right) \mathbf{J}^{\mathrm{s}}+\hat{\mathbf{n}}(\mathbf{r}) \times L^{\mathrm{s}}\left(\hat{\mathbf{n}}(\mathbf{r}) \times L^{\mathrm{v}, \mathrm{D}}\right) \mathbf{D}=\hat{\mathbf{n}}(\mathbf{r}) \times L^{\mathrm{s}}\left(-\hat{\mathbf{n}}(\mathbf{r}) \times \mathbf{E}^{\mathrm{inc}}(\mathbf{r})\right) \forall \mathbf{r} \in S, \\
L^{\mathrm{s}} \mathbf{J}^{\mathrm{s}}+L^{\mathrm{v}, \mathrm{I}} \mathbf{D}=-\mathbf{E}^{\mathrm{inc}}(\mathbf{r}) \quad \forall \mathbf{r} \in V,
\end{gathered}
$$

can be solved instead of the standard set (6) and (7).

The discretization of the operator products in (21) is by no means trivial. Various methods that use ad hoc integration rules and/or operational manipulations for discretizing the operator product $\hat{\mathbf{n}}(\mathbf{r}) \times L^{\mathrm{s}}\left(\hat{\mathbf{n}}(\mathbf{r}) \times L^{\mathrm{s}}\right)$ have been proposed [7, 9, 10]; however none of these methods is easily integrated into readily available MOM codes that discretize the standard S-EFIE using the wellknown RWG basis functions [11]. In this work, the CMP approach first proposed in [8] to discretize Calderón-preconditioned S-EFIEs is used to discretize the operator products in (21). The reader is referred to [8] for a detailed formal mathematical description of the CMP concept.

Consider the initial discretization of $S$ comprised of planar triangles on which $\mathbf{J}^{\mathrm{s}}(\mathbf{r})$ is expanded in terms of standard div-conforming RWG functions, $\mathbf{f}_{k}^{\mathrm{RWG}}(\mathbf{r}), k=1, \ldots, N^{\mathrm{RWG}} \quad$ [see (10)]. A barycentric mesh is obtained by adding the three medians to each triangle of the initial mesh; on the edges of this barycentric mesh a new set of RWG basis functions $\mathbf{f}_{k}^{\text {BRWG }}(\mathbf{r})$, 
$k=1, \ldots, N^{\mathrm{BRWG}}=6 N^{\mathrm{RWG}} \quad$ and Buffa-Christiansen $\quad(\mathrm{BC})$ basis functions $[17] \quad \mathbf{f}_{k}^{\mathrm{BC}}(\mathbf{r})$, $k=1, \ldots, N^{\mathrm{BC}}=N^{\mathrm{RWG}}\left[\right.$ Fig. 2(c)] are defined. BC basis functions $\mathbf{f}_{k}^{\mathrm{BC}}(\mathbf{r})$ are linear combinations of $\mathbf{f}_{k}^{\mathrm{BRWG}}(\mathbf{r})$ and they are div- and quasicurl-conforming. Note that $\hat{\mathbf{n}}(\mathbf{r}) \times \mathbf{f}_{k}^{\mathrm{BC}}(\mathbf{r})$ are curl- and quasidiv-conforming [Fig. 2(d)] [17]. These properties render the Gram matrix, which links spaces discretized by quasicurl-conforming $\mathbf{f}_{k}^{\mathrm{BC}}(\mathbf{r})$ and curl-conforming $\hat{\mathbf{n}}(\mathbf{r}) \times \mathbf{f}_{k}^{\mathrm{RWG}}(\mathbf{r})$, well conditioned [8]. In the operator product $\hat{\mathbf{n}}(\mathbf{r}) \times L^{\mathrm{s}}\left(\hat{\mathbf{n}}(\mathbf{r}) \times L^{\mathrm{s}}\right)$, the source and test spaces of the right S-EFIE operator $\hat{\mathbf{n}}(\mathbf{r}) \times L^{\mathrm{s}}$ are discretized using $\mathbf{f}_{k}^{\mathrm{RWG}}(\mathbf{r})$ and $\hat{\mathbf{n}}(\mathbf{r}) \times \mathbf{f}_{k}^{\mathrm{RWG}}(\mathbf{r})$, respectively. The source and test spaces of the left S-EFIE operator $\hat{\mathbf{n}}(\mathbf{r}) \times L^{\mathrm{s}}$ are discretized using $\mathbf{f}_{k}^{\mathrm{BC}}(\mathbf{r})$ and $\hat{\mathbf{n}}(\mathbf{r}) \times \mathbf{f}_{k}^{\mathrm{BC}}(\mathbf{r})$, respectively. Similarly, in the operator product $\hat{\mathbf{n}}(\mathbf{r}) \times L^{\mathrm{s}}\left(\hat{\mathbf{n}}(\mathbf{r}) \times L^{\mathrm{v}, \mathrm{D}}\right)$, the source and the test spaces of the operator $\hat{\mathbf{n}}(\mathbf{r}) \times L^{\mathrm{v}, \mathrm{D}}$ are discretized using $\mathbf{f}_{k}^{\mathrm{SWG}}(\mathbf{r})$ and $\hat{\mathbf{n}}(\mathbf{r}) \times \mathbf{f}_{k}^{\mathrm{RWG}}(\mathbf{r})$ , respectively. The left S-EFIE operator $\hat{\mathbf{n}}(\mathbf{r}) \times L^{\mathrm{s}}$ is discretized as above. These choices of basis and testing functions render the Gram matrices linking the source space of $\hat{\mathbf{n}}(\mathbf{r}) \times L^{\mathrm{s}}$ to the test spaces of $\hat{\mathbf{n}}(\mathbf{r}) \times L^{\mathrm{s}}$ and $\hat{\mathbf{n}}(\mathbf{r}) \times L^{\mathrm{v}, \mathrm{D}}$ well-conditioned. The discretized operator products are expressed as

$$
\begin{gathered}
\left\{\hat{\mathbf{n}}(\mathbf{r}) \times L^{\mathrm{s}}\left(\hat{\mathbf{n}}(\mathbf{r}) \times L^{\mathrm{s}}\right)\right\}_{\mathrm{dis}}=\mathbf{Z}^{\mathrm{BC} / \mathrm{BC}} \mathbf{G}^{-1} \mathbf{Z}^{\mathrm{RWG} / \mathrm{RWG}}, \\
\left\{\hat{\mathbf{n}}(\mathbf{r}) \times L^{\mathrm{s}}\left(\hat{\mathbf{n}}(\mathbf{r}) \times L^{\mathrm{v}, \mathrm{D}}\right)\right\}_{\mathrm{dis}}=\mathbf{Z}^{\mathrm{BC} / \mathrm{BC}} \mathbf{G}^{-1} \mathbf{Z}^{\mathrm{RWG} / \mathrm{SWG}} .
\end{gathered}
$$

The entries of the impedance matrices $\mathbf{Z}^{\mathrm{RWG} / \mathrm{RWG}}$ and $\mathbf{Z}^{\mathrm{RWG} / \mathrm{SWG}}$ are given by (16) and (18) while those of the impedance matrix $\mathbf{Z}^{\mathrm{BC} / \mathrm{BC}}$ and Gram matrix $\mathbf{G}$ are

$$
\mathbf{Z}_{k, k^{\prime}}^{\mathrm{BC} / \mathrm{BC}}=\left\langle\hat{\mathbf{n}}(\mathbf{r}) \times \mathbf{f}_{k}^{\mathrm{BC}}(\mathbf{r}), \hat{\mathbf{n}}(\mathbf{r}) \times L^{\mathrm{s}} \mathbf{f}_{k^{\prime}}^{\mathrm{BC}}\right\rangle_{\mathrm{s}},
$$

and

$$
\mathbf{G}_{k, k^{\prime}}=\left\langle\hat{\mathbf{n}}(\mathbf{r}) \times \mathbf{f}_{k}^{\mathrm{RWG}}(\mathbf{r}), \mathbf{f}_{k^{\prime}}^{\mathrm{BC}}(\mathbf{r})\right\rangle_{\mathrm{s}} .
$$

Note that if one would use div- and curl-conforming RWGs to discretize the source and test spaces of $\hat{\mathbf{n}}(\mathbf{r}) \times L^{\mathrm{s}}$ and test space of $\hat{\mathbf{n}}(\mathbf{r}) \times L^{\mathrm{v}, \mathrm{D}}$, the Gram matrix $\mathbf{G}_{k, k^{\prime}}=\left\langle\hat{\mathbf{n}}(\mathbf{r}) \times \mathbf{f}_{k}^{\mathrm{RWG}}(\mathbf{r}), \mathbf{f}_{k^{\prime}}^{\mathrm{RWG}}(\mathbf{r})\right\rangle_{\mathrm{s}}$ would be singular [8]. Let $X^{\mathrm{RWG}}, X^{\mathrm{BRWG}}$, and $X^{\mathrm{BC}}$ denote the spaces spanned by $\mathbf{f}_{k}^{\mathrm{RWG}}(\mathbf{r}), \mathbf{f}_{k}^{\mathrm{BRWG}}(\mathbf{r})$, and $\mathbf{f}_{k}^{\mathrm{BC}}(\mathbf{r})$, respectively. Upon constructing transformation matrices $\mathbf{P}$ and $\mathbf{R}$ that express basis functions in $X^{\mathrm{BC}}$ and $X^{\mathrm{RWG}}$ as linear combinations of those in $X^{\text {BRWG }},(23)$ can be rewritten using only two impedance matrices of the same type, viz. $\mathbf{Z}^{\text {BRWG/BRWG }}$, as

$$
\left\{\hat{\mathbf{n}}(\mathbf{r}) \times L^{\mathrm{s}}\left(\hat{\mathbf{n}}(\mathbf{r}) \times L^{\mathrm{s}}\right)\right\}_{\mathrm{dis}}=\mathbf{P}^{T} \mathbf{Z}^{\mathrm{BRWG} / \mathrm{BRWG}}\left(\mathbf{P G}^{-1} \mathbf{R}^{\mathrm{T}}\right) \mathbf{Z}^{\mathrm{BRWG} / \mathrm{BRWG}} \mathbf{R},
$$


where and the entries of the impedance matrix $\mathbf{Z}^{\text {BRWG/BRWG }}$ are

$$
\mathbf{Z}_{k, k^{\prime}}^{\mathrm{BRWG} / \mathrm{BRWG}}=\left\langle\hat{\mathbf{n}}(\mathbf{r}) \times \mathbf{f}_{k}^{\mathrm{BRWG}}(\mathbf{r}), \hat{\mathbf{n}}(\mathbf{r}) \times L^{\mathrm{s}} \mathbf{f}_{k^{\prime}}^{\mathrm{BRWG}}\right\rangle_{\mathrm{s}} .
$$

Because only the barycentric mesh (and not the initial one) is supplied to the code, (24) is replaced with the discretization

$$
\left\{\hat{\mathbf{n}}(\mathbf{r}) \times L^{\mathrm{s}}\left(\hat{\mathbf{n}}(\mathbf{r}) \times L^{\mathrm{v}, \mathrm{D}}\right)\right\}_{\mathrm{dis}}=\mathbf{P}^{T} \mathbf{Z}^{\mathrm{BRWG} / \mathrm{BRWG}}\left(\mathbf{P G}^{-1} \mathbf{R}^{\mathrm{T}}\right) \mathbf{Z}^{\mathrm{BRWG} / \mathrm{SWG}},
$$

where the entries of the impedance matrix $\mathbf{Z}^{\mathrm{BRWG} / \mathrm{SWG}}$ are

$$
\mathbf{Z}_{k, k^{\prime}}^{\mathrm{BRWG} / S W G}=\left\langle\hat{\mathbf{n}}(\mathbf{r}) \times \mathbf{f}_{k}^{\mathrm{BRWG}}(\mathbf{r}), L^{\mathrm{v}, \mathrm{D}} \mathbf{f}_{k^{\prime}}^{\mathrm{SWG}}\right\rangle_{\mathrm{v}} .
$$

The discretization of the operator $L^{\mathrm{s}}$ in (22) can be achieved classically using $\mathbf{f}_{k}^{\mathrm{RWG}}(\mathbf{r})$ and $\chi(\mathbf{r}) \mathbf{f}_{k}^{\mathrm{SWG}}(\mathbf{r})$. However this is not done directly but using $\mathbf{f}_{k}^{\mathrm{BRWG}}(\mathbf{r})$ and $\chi(\mathbf{r}) \mathbf{f}_{k}^{\mathrm{SWG}}(\mathbf{r})$ since only the barycentric mesh (and not the initial one) is supplied to the code. In other words,

$$
\left\{L^{\mathrm{s}}\right\}_{\mathrm{dis}}=\mathbf{Z}^{\mathrm{SWG} / \mathrm{RWG}}=\mathbf{Z}^{\mathrm{SWG} / \mathrm{BRWG}} \mathbf{R},
$$

where the entries of the impedance matrix $\mathbf{Z}^{\mathrm{SWG} / \text { BRWG }}$ are given by

$$
\mathbf{Z}_{k, k^{\prime}}^{\mathrm{SWG} / \mathrm{BRWG}}=\left\langle\chi(\mathbf{r}) \mathbf{f}_{k}^{\mathrm{SWG}}(\mathbf{r}), L^{\mathrm{s}} \mathbf{f}_{k^{\prime}}^{\mathrm{BRWG}}\right\rangle_{\mathrm{v}} .
$$

The discretization of the operator $L^{\mathrm{v}, \mathrm{I}}$ in (22) is unchanged:

$$
\left\{L^{\mathrm{v}, \mathrm{I}}\right\}_{\mathrm{dis}}=\mathbf{Z}^{\mathrm{SWG} / \mathrm{SWG}} \text {. }
$$

The entries of the impedance matrix $\mathbf{Z}^{\text {SWG/SRWG }}$ are given by (19).

The impedance matrices $\mathbf{Z}^{\text {BRWG/BRWG }}, \mathbf{Z}^{\text {BRWG/SWG }}, \mathbf{Z}^{\text {SWG/BRWG }}$, and $\mathbf{Z}^{\text {SWG/SRWG }}$ are trivially computed using existing MOM codes that use RWG and SWG basis functions. Explicit expressions of the elements of the matrices $\mathbf{P}$ and $\mathbf{R}$ can be found in [8].

Inserting (27), (29), (31), and (33) into (21) and (22) discretizing the right hand sides, rearranging the resulting equations, and applying diagonal preconditioning yields

$$
\begin{gathered}
{\left[\begin{array}{ll}
\mathbf{D}^{\mathrm{BRWG}} & \mathbf{0} \\
\mathbf{0} & \mathbf{D}^{\mathrm{SWG}}
\end{array}\right]\left(\left[\begin{array}{cc}
\mathbf{P}^{\mathrm{T}} & \mathbf{0} \\
\mathbf{0} & \mathbf{0}
\end{array}\right] \mathbf{Z}^{\mathrm{B}}\left[\begin{array}{ll}
\mathbf{P} & \mathbf{0} \\
\mathbf{0} & \mathbf{0}
\end{array}\right]+\left[\begin{array}{ll}
\mathbf{0} & \mathbf{0} \\
\mathbf{0} & \mathbf{1}
\end{array}\right]\right)\left[\begin{array}{ll}
\mathbf{G} & \mathbf{0} \\
\mathbf{0} & \mathbf{1}
\end{array}\right]^{-1}\left(\left[\begin{array}{cc}
\mathbf{R}^{\mathrm{T}} & \mathbf{0} \\
\mathbf{0} & \mathbf{1}
\end{array}\right] \mathbf{Z}^{\mathrm{B}}\left[\begin{array}{cc}
\mathbf{R} & \mathbf{0} \\
\mathbf{0} & \mathbf{1}
\end{array}\right]\right) \mathbf{I}=} \\
{\left[\begin{array}{ll}
\mathbf{D}^{\mathrm{BRWG}} & \mathbf{0} \\
\mathbf{0} & \mathbf{D}^{\mathrm{SWG}}
\end{array}\right]\left(\left[\begin{array}{cc}
\mathbf{P}^{\mathrm{T}} & \mathbf{0} \\
\mathbf{0} & \mathbf{0}
\end{array}\right] \mathbf{Z}^{\mathrm{B}}\left[\begin{array}{ll}
\mathbf{P} & \mathbf{0} \\
\mathbf{0} & \mathbf{0}
\end{array}\right]+\left[\begin{array}{ll}
\mathbf{0} & \mathbf{0} \\
\mathbf{0} & \mathbf{1}
\end{array}\right]\right)\left[\begin{array}{cc}
\mathbf{G} & \mathbf{0} \\
\mathbf{0} & \mathbf{1}
\end{array}\right]^{-1}\left[\begin{array}{cc}
\mathbf{R}^{\mathrm{T}} & \mathbf{0} \\
\mathbf{0} & \mathbf{1}
\end{array}\right] \mathbf{V}^{\mathrm{B}}}
\end{gathered}
$$

Here, the impedance matrix $\mathbf{Z}^{\mathrm{B}}$ is

$$
\mathbf{Z}^{\mathrm{B}}=\left[\begin{array}{cc}
\mathbf{Z}^{\text {BRWG/BRWG }} & \mathbf{Z}^{\text {BRWG/SWG }} \\
\mathbf{Z}^{\text {SWG/BRWG }} & \mathbf{Z}^{\text {SWG/SWG }}
\end{array}\right],
$$


the entries of the matrices $\mathbf{D}^{\mathrm{BRWG}}$ and $\mathbf{D}^{\mathrm{SWG}}$ are

$$
\begin{aligned}
\mathbf{D}_{k, k^{\prime}}^{\mathrm{SWG}} & =\left\{\begin{array}{ll}
1 / \mathbf{Z}_{k, k^{\prime}}^{\mathrm{SWG},} & k=k^{\prime} \\
0 & \text { else }
\end{array},\right. \\
\mathbf{D}_{k, k^{\prime}}^{\mathrm{BRW}} & =\left\{\begin{array}{ll}
1 / \mathbf{G}_{k, k^{\prime}}, & k=k^{\prime} \\
0 & \text { else }
\end{array},\right.
\end{aligned}
$$

the entries of the right hand side vector $\mathbf{V}^{\mathrm{B}}$ are

$$
\mathbf{V}_{\mathrm{k}}^{\mathrm{B}}=\left\{\begin{array}{ll}
-\left\langle\hat{\mathbf{n}}(\mathbf{r}) \times \mathbf{f}_{k}^{\mathrm{BRWG}}(\mathbf{r}), \hat{\mathbf{n}}(\mathbf{r}) \times \mathbf{E}^{\mathrm{inc}}(\mathbf{r})\right\rangle_{\mathrm{s}}, & k \leq N^{\mathrm{BRWG}} \\
-\left\langle\chi(\mathbf{r}) \mathbf{f}_{k-N^{\mathrm{BRWG}}}^{\mathrm{SWG}}(\mathbf{r}), \mathbf{E}^{\mathrm{inc}}(\mathbf{r})\right\rangle_{\mathrm{v}}, & \text { else }
\end{array},\right.
$$

and $\mathbf{I}$ is the vector of unknown coefficients [see (13)]. Note that multiplication with $\mathbf{D}^{\text {SWG }}$ properly scales the entries of $\mathbf{D}^{\text {SWG }} \mathbf{Z}^{\text {SWG }}$ and multiplication with $\mathbf{D}^{\text {BRWG }}$ renders $\mathbf{D}^{\mathrm{BRWG}} \mathbf{Z}^{\mathrm{BC} / \mathrm{BC}} \mathbf{G}^{-1} \mathbf{Z}^{\mathrm{RWG} / \mathrm{RWG}}$ well-conditioned [8] in the presence of multiscale discretizations. Matrix equation (34) is solved for I iteratively; the number of iterations is independent of the smallest edge sizes $\delta^{\mathrm{s}}$ and $\delta^{\mathrm{v}}$ as demonstrated in Section III. The computational cost of solving (34) is that of performing the matrix-vector multiplications on its left hand side, times the number of iterations. The cost of multiplying the sparse matrices $\mathbf{P}, \mathbf{R}$, and $\mathbf{G}$ by a vector scales as only $O\left(N^{\mathrm{RWG}}\right)$. (Note that inversion of the sparse Gram matrix $\mathbf{G}$ is never carried out explicitly; whenever the matrix-vector product $\mathbf{G}^{-1} \mathbf{y}$ is needed, it is computed via the iterative solution of the linear system $\mathbf{D}^{\mathrm{BRWG}} \mathbf{G} \mathbf{x}=\mathbf{D}^{\mathrm{BRWG}} \mathbf{y}$, which only requires a few iterations [8].) The dominant computational cost at a given iteration is then due to the multiplication of $\mathbf{Z}^{\mathrm{B}}$ with a vector, which can be reduced using various acceleration techniques [13-15]. In this work, AIM [13] is used to for this purpose. Let $C$ and $C^{\mathrm{B}}$ represent the costs of multiplying $\mathbf{Z}$ and $\mathbf{Z}^{\mathrm{B}}$ by a vector; then $C^{\mathrm{CMP}} / C=\left[N_{\mathrm{c}}^{\mathrm{CMP}} \log \left(N_{\mathrm{c}}^{\mathrm{CMP}}\right)\right] /\left[N_{\mathrm{c}} \log \left(N_{\mathrm{c}}\right)\right], \quad$ where $\quad N_{\mathrm{c}} \propto 1 /\left(\Delta_{\mathrm{c}}\right)^{3} \quad$ and $N_{\mathrm{c}}^{\mathrm{CMP}} \propto 1 /\left(\Delta_{\mathrm{c}}^{\mathrm{CMP}}\right)^{3}$ are the numbers of nodes on the three dimensional (3-D) auxiliary AIM grid that encloses the composite structure and is used for accelerating the multiplication of $\mathbf{Z}$ and $\mathbf{Z}^{\mathrm{B}}$ by a vector, respectively. Here, $\Delta_{\mathrm{c}}$ and $\Delta_{\mathrm{c}}^{\mathrm{CMP}}$ are the AIM grid sizes (i.e. AIM node spacing); they are assumed identical along the $x, y$, and $z$ directions. As a rule of thumb, $\Delta_{\mathrm{c}} \cong \delta_{\mathrm{c}}^{\text {ave }}$ and $\Delta_{\mathrm{c}}^{\mathrm{CMP}} \cong \delta_{\mathrm{c}}^{\mathrm{CMP} \text {,ave }}$, where $\delta_{\mathrm{c}}^{\text {ave }}$ and $\delta_{\mathrm{c}}^{\mathrm{CMP} \text {,ave }}$ are the average edge sizes in the (combined) initial surface and volume meshes, and the (combined) barycentric surface and volume meshes, respectively. Ignoring the cost of operations associated with the multiplication of the sparse matrices, the total cost of solving (12) is $C_{\text {Tот }}=N_{\text {iter }} C$, while the cost of solving (34) is $C_{\mathrm{TOT}}^{\mathrm{CMP}}=2 N_{\text {iter }}^{\mathrm{CMP}} C^{\mathrm{CMP}}$. Here $N_{\text {iter }}^{\mathrm{CMP}}$ and $N_{\text {iter }}$ are the numbers of iterations required for the relative 
residual error of the solutions of (12) and (34) to reach a certain treshhold. By comparing $C_{\mathrm{TOT}}^{\mathrm{CMP}}$ to $C_{\mathrm{TOT}}$, it is concluded that the iterative solution of (34) will be faster than that of (12) as long as $N_{\text {iter }}^{\text {CMP }}<0.5 N_{\text {iter }}\left(\delta_{\mathrm{c}}^{\text {CMP,ave }} / \delta_{\mathrm{c}}^{\text {ave }}\right)^{3} \log \left(\delta_{\mathrm{c}}^{\text {CMP,ave }}\right) / \log \left(\delta_{\mathrm{c}}^{\text {ave }}\right)$. The ratio $\delta_{\mathrm{c}}^{\text {CMP,ave }} / \delta_{\mathrm{c}}^{\text {ave }}$ is always smaller than 1 (because of the barycentric division of the surface discretization) but typically larger than 0.5 (because of the presence of the volumetric mesh).

A more efficient but less trivial implementation is possible. Inserting (23), (24), (31) and (33) into (21) and (22), discretizing the right hand sides, and applying diagonal preconditioning yields

$$
\left[\begin{array}{ll}
\mathbf{D}^{\mathrm{BRWG}} & \mathbf{0} \\
\mathbf{0} & \mathbf{D}^{\mathrm{SWG}}
\end{array}\right]\left[\begin{array}{cc}
\mathbf{Z}^{\mathrm{BC} / \mathrm{BC}} & \mathbf{0} \\
\mathbf{0} & \mathbf{1}
\end{array}\right]\left[\begin{array}{ll}
\mathbf{G} & \mathbf{0} \\
\mathbf{0} & \mathbf{1}
\end{array}\right]^{-1} \mathbf{Z I}=\left[\begin{array}{ll}
\mathbf{D}^{\mathrm{BRWG}} & \mathbf{0} \\
\mathbf{0} & \mathbf{D}^{\mathrm{SWG}}
\end{array}\right]\left[\begin{array}{cc}
\mathbf{Z}^{\mathrm{BC} / \mathrm{BC}} & \mathbf{0} \\
\mathbf{0} & \mathbf{1}
\end{array}\right]\left[\begin{array}{ll}
\mathbf{G} & \mathbf{0} \\
\mathbf{0} & \mathbf{1}
\end{array}\right]^{-1} \mathbf{V} .
$$

Solution of (39) using an existing MOM code is far less trivial than that of (34) since now $\mathbf{Z}^{\mathrm{BC} / \mathrm{BC}}$ needs to be computed explicitly. Additionally, one needs to modify the AIM accelerator to allow for the fast multiplication of $\mathbf{Z}^{\mathrm{BC} / \mathrm{BC}}$ by a vector. Assuming such an $\mathrm{AIM}$ accelerator is constructed, it can use the same auxiliary grid used for accelerating the matrix-vector multiplication associated with $\mathbf{Z}$ since the support of $\mathrm{BC}$ basis functions is (roughly) the same as that of RWG basis functions defined on the standard mesh (i.e. node spacing needed for auxiliary AIM grids is the same). This means that the cost of solving (39) is $C_{\mathrm{TOT}}^{\mathrm{CMP}}=\left[N_{\text {iter }}^{\mathrm{CMP}} / N_{\text {iter }}\right] \gamma C_{\mathrm{TOT}}$, where $\gamma$ depends on what ratio of the AIM's grid encloses the PEC surfaces; $\gamma$ is at most 2 (This happens when the structure consists of only PEC surfaces). It is clear from this discussion that the iterative solution of (39) is faster than that of (12) as long as $N_{\text {iter }}^{\mathrm{CMP}}<\gamma N_{\text {iter }}$ is satisfied. Numerical results show that this is satisfied even for moderately dense discretizations.

\section{Numerical Results}

In this section, the proposed method is applied to the analysis of scattering from spheres and a shuttle loaded with a dielectric-filled waveguide slot antenna, and radiation from dielectric antennas inclusive their PEC feeds. The results presented here were obtained using a parallel and AIM-accelerated MOM code that uses a transpose-free quasi-minimal residual iterative scheme [18] to solve matrix equations (34) and (12); a diagonal preconditioner is used for (12). All simulations were carried out on a cluster of dual-core 2.8-GHz AMD Opteron $2220 \mathrm{SE}$ processors at the Center for Advanced Computing, University of Michigan.

\section{A. Scattering from Spheres}

\section{PEC and Dielectric Spheres}


Consider the two adjacent spheres, one PEC and the other dielectric, shown in Fig. 3(a). The PEC and dielectric spheres are centered about the origin and $(2.5 \mathrm{~m}, 0,0)$, respectively; both spheres have radius $1 \mathrm{~m}$. The dielectric constant of the dielectric sphere is $\varepsilon(\mathbf{r})=4.0 \varepsilon_{0}$. The spheres are excited by a $\hat{\mathbf{y}}$ polarized plane wave propagating in the $\hat{\mathbf{z}}$ direction. The frequency of excitation is $f=15 \mathrm{MHz}(\lambda=19.986 \mathrm{~m})$. The simulation is repeated for seven different discretizations with smallest edge sizes ranging from $\delta^{\mathrm{s}}=\delta^{\mathrm{v}}=14.383 \mathrm{~cm}$ to $\delta^{\mathrm{s}}=\delta^{\mathrm{v}}=1.1264 \mathrm{~cm}$. Table I presents the number of RWGs $\left(N^{\mathrm{RWG}}\right)$ and SWGs $\left(N^{\mathrm{SWG}}\right)$ for all models analyzed. Fig. 3(b) presents the number of iterations required (for the relative residual error of the solutions of (34) and (12) to reach $10^{-6}$ ) versus $\delta^{\mathrm{s}}=\delta^{\mathrm{v}}$. As expected, the number of iterations required for the solution of (34) is independent of the discretization density; it is roughly 25 . For the simulation with the densest discretization density $\left(\delta^{\mathrm{s}}=\delta^{\mathrm{v}}=1.1264 \mathrm{~cm}\right)$ measured CPU times indicated that the iterative solution of (34) was approximately 6.9 times faster than that of (12). Fig. 3(c) presents the spheres' radar cross sections (RCSs) computed on the $\phi=0^{\circ}$ and $\phi=90^{\circ}$ planes after solving (34) or (12) using the $\delta^{\mathrm{s}}=\delta^{\mathrm{v}}=4.7456 \mathrm{~cm}$ mesh. The relative L2 norms of the difference between the RCS results on the $\phi=0^{\circ}$ and $\phi=90^{\circ}$ planes are $0.0221 \%$ and $0.0164 \%$, respectively.

\section{Dielectric Coated PEC Sphere}

The proposed method is used to analyze scattering from a dielectric-coated PEC sphere centered at the origin; the radius of the sphere and the thickness of the dielectric shell are $1 \mathrm{~m}$ and $0.2 \mathrm{~m}$, respectively [Fig. 4(a)]. The dielectric constant of the shell is $\varepsilon(\mathbf{r})=4.0 \varepsilon_{0}$. The sphere is excited by the plane wave used in Section II-A.I. Similarly, the simulation is repeated for seven different discretizations with smallest edge sizes changing from $\delta^{\mathrm{s}}=\delta^{\mathrm{v}}=14.383 \mathrm{~cm}$ and $\delta^{\mathrm{s}}=\delta^{\mathrm{v}}=1.1264 \mathrm{~cm}$. Table II presents $N^{\mathrm{RWG}}$ and $N^{\mathrm{SWG}}$ for all discretizations. Fig. 4(b) presents the number of iterations required (for the relative error of the solutions of (34) and (12) to reach $10^{-6}$ ) versus $\delta^{\mathrm{s}}=\delta^{\mathrm{v}}$. The number of iterations required for the solution of (34) is constant and hovers around 24. For the simulation with the densest discretization density ( $\delta^{\mathrm{s}}=\delta^{\mathrm{v}}=1.1264 \mathrm{~cm}$ ) measured CPU times indicated that the iterative solution of (34) was approximately 11.2 times faster than that of (12). Fig. 4(c) shows that the solutions of (34) and (12) for the simulation with $\delta^{s}=\delta^{v}=6.9214 \mathrm{~cm}$ are practically the same; the relative norm of the difference between both solutions is $0.1874 \%$.

\section{B. Scattering from a Space Shuttle Model}


The proposed technique is used to analyze low-frequency scattering from a shuttle model with a dielectric-filled slot waveguide mounted on its side. The shuttle model is excited by a $\hat{\mathbf{x}}$ polarized plane wave propagating in the $-\hat{\mathbf{z}}$ direction at $f=26.4 \mathrm{MHz}(\lambda=11.356 \mathrm{~m})$. The length, width, and height of the shuttle are $3.6520 \lambda, 2.3445 \lambda$, and $1.3989 \lambda$, respectively [Fig. 5(a)]. A slot-waveguide antenna, which is filled with dielectric with $\varepsilon(\mathbf{r})=4.0 \varepsilon_{0}$, is located on the side of the shuttle [Fig. 5(b)]. The width and height of the slot and the length of the waveguide are $\lambda / 150.09, \lambda / 32.792$, and $\lambda / 50.032$, respectively. The multiscale discretization of the shuttle and the waveguide surfaces is shown in Fig. 5(c). For this mesh the largest, average, and the smallest element sizes are $1.1400 \mathrm{~m}(\lambda / 9.96108), 0.35120 \mathrm{~m}(\lambda / 32.3339), 1.0589 \mathrm{~cm}($ $\lambda / 1072.45$ ), respectively; also $N^{\mathrm{RWG}}=29409$, and $N^{\mathrm{SWG}}=5022$. The iterative solver required 274 and 7396 iterations for the relative residual error of the solutions of (34) and (12) to reach $10^{-6}$, respectively [Fig. 5(d)]. Measured CPU times indicated that the iterative solution of (34) was approximately 2.4 times faster than that of (12). The relative L2 norm of the difference between the two solutions is $0.2168 \%$. Fig. 5(d) shows three different views of the magnitude of the current induced on the shuttle's surface.

\section{Radiation from a Hemispherical Dielectric Resonator}

Consider the hemispherical dielectric resonator antenna (with an air gap) shown in Figs. 6 (a)-(c). The dielectric constant of the hemispherical shell is $\varepsilon(\mathbf{r})=8.9 \varepsilon_{0}$. The resonator is excited by a feed probe at $f=2.0 \mathrm{GHz}(\lambda=0.14989 \mathrm{~m})$. The multiscale nature of the spatial mesh around the feed probe is highlighted in Fig. 6 (d). The fine discretization around the feed is called for to properly model the curvature of the feed probe and the distribution of fields around it. For this discretization, the largest, average, and the smallest element sizes are $4.46694 \mathrm{~mm}(\lambda / 33.5567)$, $1.11553 \mathrm{~mm}(\lambda / 134.372)$, and $0.124458 \mathrm{~mm}(\lambda / 1204.39)$, respectively; also $N^{\mathrm{RWG}}=43943$ and $N^{\text {SWG }}=166953$. The iterative solver required 737 and 11675 iterations for the relative residual error of the solutions of (34) and (12) to reach $10^{-6}$, respectively [Fig. 6 (e)]. Measured CPU times indicated that the iterative solution of (34) was approximately 3.6 times faster than that of (12). The relative L2 norm of the difference between the two solutions is $0.8325 \%$. Fig. 6 (e) shows the normalized radiated field patterns (on the $x z$ and $y z$ planes) obtained from the solutions of (12) and (34).

\section{Radiation from a Dielectric Rod Antenna}


Finally, the proposed method is used to analyze radiation from a dielectric rod antenna [Figs. 7 (a)-(d) [19], with $\varepsilon(\mathbf{r})=2.1 \varepsilon_{0}$. The end of the rod is coated with an antireflective dielectric layer, with $\varepsilon(\mathbf{r})=1.45 \varepsilon_{0}$. The antenna is fed by a rectangular PEC waveguide and the waveguide is excited by a feed probe at $f=9.0 \mathrm{GHz}(\lambda=3.33102 \mathrm{~cm})[19]$. Similar to the previous example, the surface of the feed probe and the waveguide surfaces near to the probe are densely discretized. For this simulation the largest, average, and smallest element sizes are $2.89224 \mathrm{~mm}$ ( $\lambda / 11.5171), 1.48322 \mathrm{~mm}(\lambda / 22.4579)$, and $0.044623 \mathrm{~mm}(\lambda / 746.480)$, respectively; also $N^{\text {RWG }}=44692$ and $N^{\text {SWG }}=293737$. The iterative solver required 659 and 14633 iterations for the relative residual error of the solutions of (34) and (12) to reach $10^{-6}$, respectively [Fig. 7 (e)]. Measured CPU times indicated that the iterative solution of (34) was approximately 6.5 times faster than that of (12). The relative norm of the difference between the two solutions is $0.9152 \%$. Fig. 7 (f) shows that the normalized radiated field pattern (on $x z$ plane) obtained from the solution of (34) agrees well with that computed using the imaginary-distance beampropagation method [19].

\section{Conclusion}

This paper presented a CMP-based regularizer for a coupled set of S- and V-EFIEs pertinent to the analysis of densely discretized hybrid PEC-dielectric structures. The proposed technique combines a CMP for the S-EFIE and a diagonal preconditioner for the V-EFIE. Just like in the original CMP, the preconditioner presented herein is multiplicative and easily integrated into available MOM codes that discretize S- and V-EFIEs using RWG and SWG basis functions, respectively. The proposed preconditioner is used in conjunction with an existing parallel and AIM accelerated MOM code. The numerical results obtained using this code confirmed the effectiveness of the proposed technique and its applicability to the electromagnetic characterization of composite structures with sub-wavelength features.

\section{Acknowledgments}

Authors would like to thank Mr. Felipe Valdés for his help in preparing meshes for the hemispherical dielectric resonator and the dielectric rod antenna.

This work was supported by the Air Force Office of Scientific Research under Grant MURI F014432051936 and by the National Science Foundation under Grant DMS 0713771. 


\section{Figure Captions}

Figure 1: Description of scattering problem involving an abstract composite structure.

Figure 2: Basis functions used in the discretization of S-EFIE. (a) Div-conforming RWG basis function, $\mathbf{f}_{k}^{\mathrm{RWG}}(\mathbf{r})$, (b) curl-conforming RWG basis function, $\hat{\mathbf{n}}(\mathbf{r}) \times \mathbf{f}_{k}^{\mathrm{RWG}}(\mathbf{r})$, (c) div- and quasicurl-conforming BC basis function $\mathbf{f}_{k}^{\mathrm{BC}}(\mathbf{r})$, (d) curl- and quasidiv-conforming $\mathrm{BC}$ function, $\hat{\mathbf{n}}(\mathbf{r}) \times \mathbf{f}_{k}^{\mathrm{BC}}(\mathbf{r})$.

Figure 3: Analysis of scattering from PEC and dielectric spheres. (a) Geometry and excitation description. (b) Number of iterations required for the relative residual error of the solutions of (34) and (12) to reach $10^{-6}$, versus $\delta^{\mathrm{s}}=\delta^{\mathrm{v}}$. (c) Comparison of RCS obtained on $\phi=0^{\circ}$ and $\phi=90^{\circ}$ planes after solving (34) and (12).

Figure 4: Analysis of scattering from a dielectric-coated PEC sphere. (a) Geometry and excitation description. (b) Number of iterations required for the relative residual error of the solutions of (34) and (12) to reach $10^{-6}$, versus $\delta^{\mathrm{s}}=\delta^{\mathrm{v}}$. (c) Comparison of solutions of (34) and (12).

Figure 5: Analysis of scattering from a shuttle model with a dielectric-filled slot waveguide mounted on its fuselage. (a) Geometry and excitation description. (b) The multiscale discretization of the shuttle and the waveguide surfaces. (c) Relative residual error obtained during the iterative solution of (34) and (12). (d) Current density induced on surfaces of the shuttle and the waveguide decibel scale.

Figure 6: Analysis of radiation from a hemispherical dielectric resonator. View of the geometry from (a) top and (b) bottom. (c) Cross section of the geometry (dimensions are in $\mathrm{cm}$ ). (d) Multiscale surface mesh of the geometry (zoomed to the feed probe). (e) Relative residual error obtained during the iterative solution of (34) and (12). (f) Normalized field patterns computed on $x z$ and $y z$ planes.

Figure 7: Analysis of radiation from a dielectric rod antenna. (a) Isometric view of the whole geometry. (b) View of the metallic feed (dimensions are in $\mathrm{mm}$ ). (c) View of the antireflective dielectric layer (dimensions are in $\mathrm{mm}$ ). (d) Cross section of the whole geometry (dimensions are in $\mathrm{mm}$ ). (e) Relative residual error obtained during the iterative solution of (34) and (12). (f) Normalized field patterns computed on $x z$ plane. 


\section{Tables}

Table I. The numbers of standard RWGs $\left(N^{\mathrm{RWG}}\right)$ and SWGs $\left(N^{\mathrm{SWG}}\right)$ for all seven discretizations.

\begin{tabular}{c|c|c}
$\delta^{\mathrm{s}}=\delta^{\mathrm{v}}(\mathrm{cm})$ & $N^{\mathrm{RWG}}$ & $N^{\mathrm{SWG}}$ \\
\hline 14.383 & 1062 & 4634 \\
\hline 11.092 & 1926 & 9786 \\
\hline 6.9214 & 3960 & 24082 \\
\hline 4.7456 & 7632 & 54232 \\
\hline 2.9893 & 16560 & 135677 \\
\hline 1.7720 & 67464 & 652352 \\
\hline 1.1264 & 136923 & 1393460
\end{tabular}

Table II. The numbers of standard RWGs $\left(N^{\mathrm{RWG}}\right)$ and SWGs $\left(N^{\mathrm{SWG}}\right)$ for all seven discretizations.

\begin{tabular}{c|c|c}
$\delta^{\mathrm{s}}=\delta^{\mathrm{v}}(\mathrm{cm})$ & $N^{\mathrm{RWG}}$ & $N^{\mathrm{SWG}}$ \\
\hline 14.383 & 1062 & 5962 \\
\hline 11.092 & 1926 & 11172 \\
\hline 6.9214 & 3960 & 37014 \\
\hline 4.7456 & 7632 & 87324 \\
\hline 2.9893 & 16560 & 261954 \\
\hline 1.7720 & 67464 & 1419176 \\
\hline 1.1264 & 136923 & 2747863
\end{tabular}




\section{Figures}

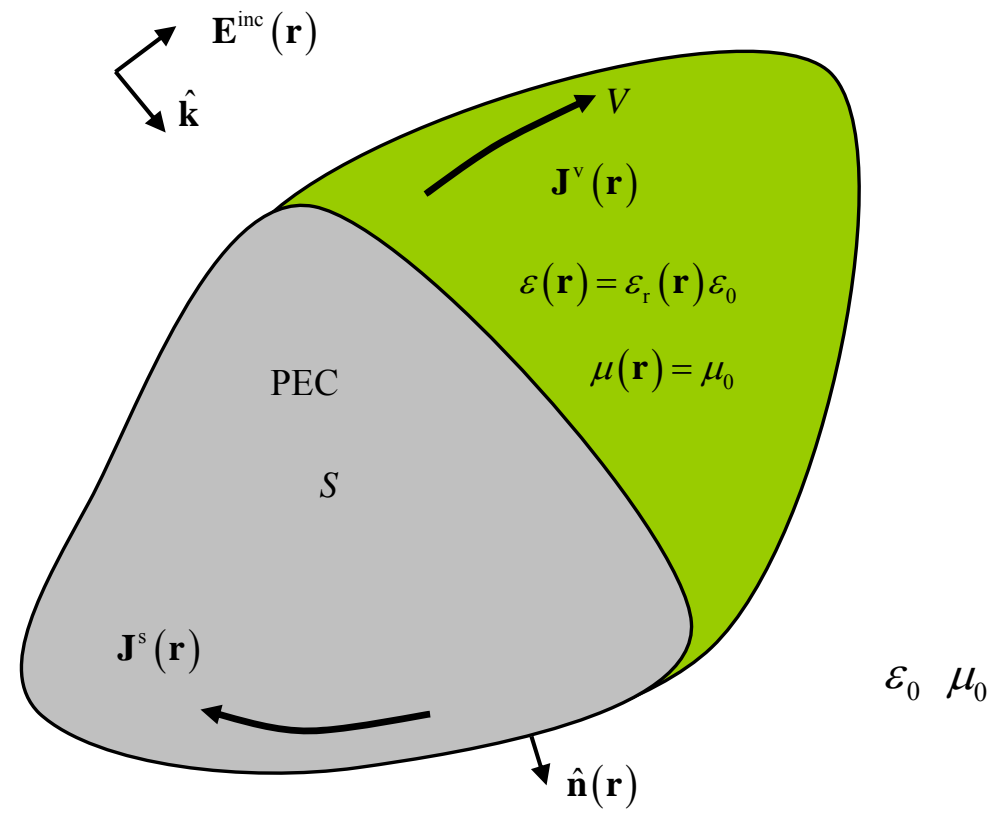

Figure 1

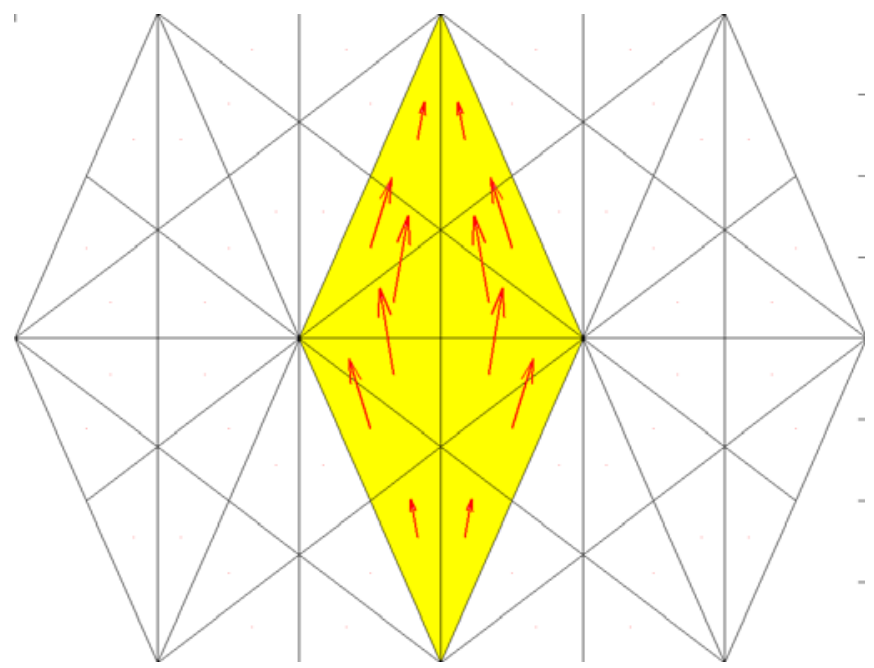

Figure 2(a) 


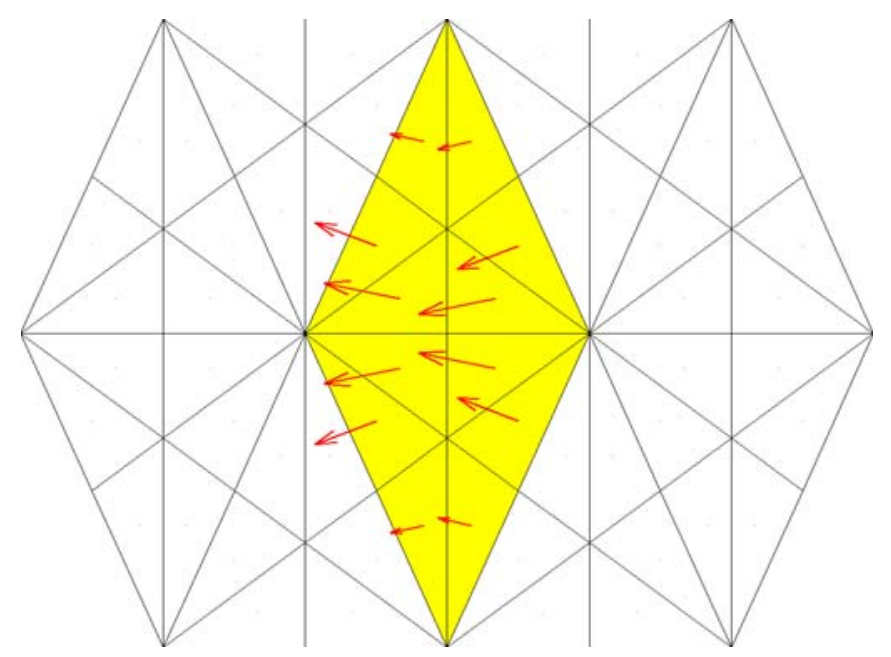

Figure 2(b)

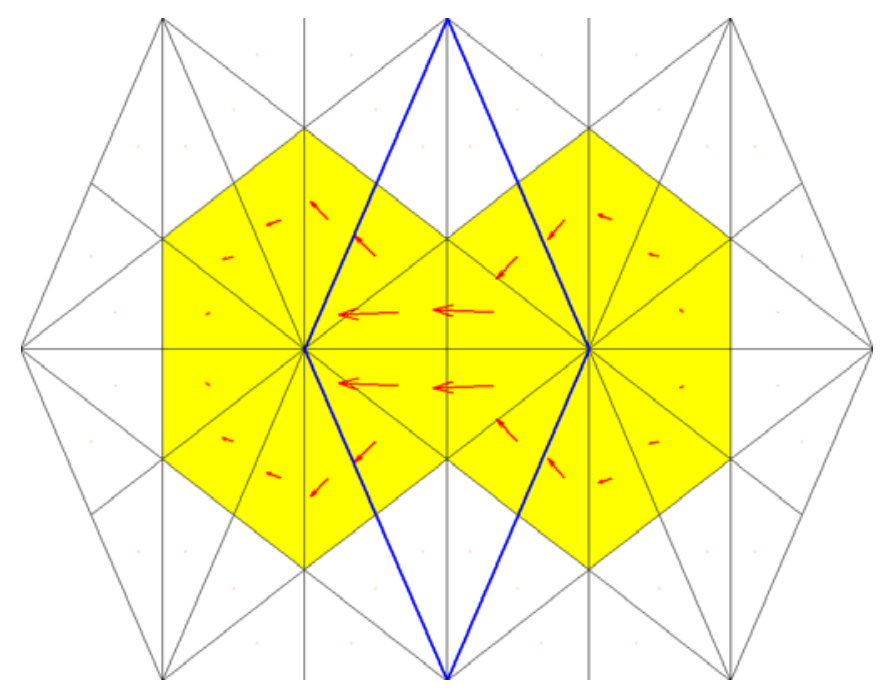

Figure 2(c) 


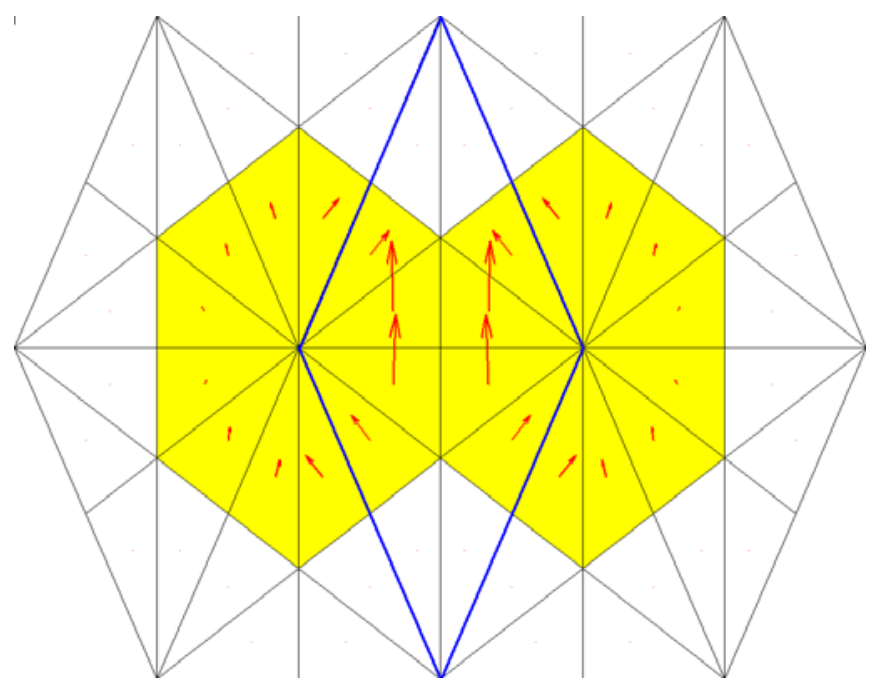

Figure 2(d)

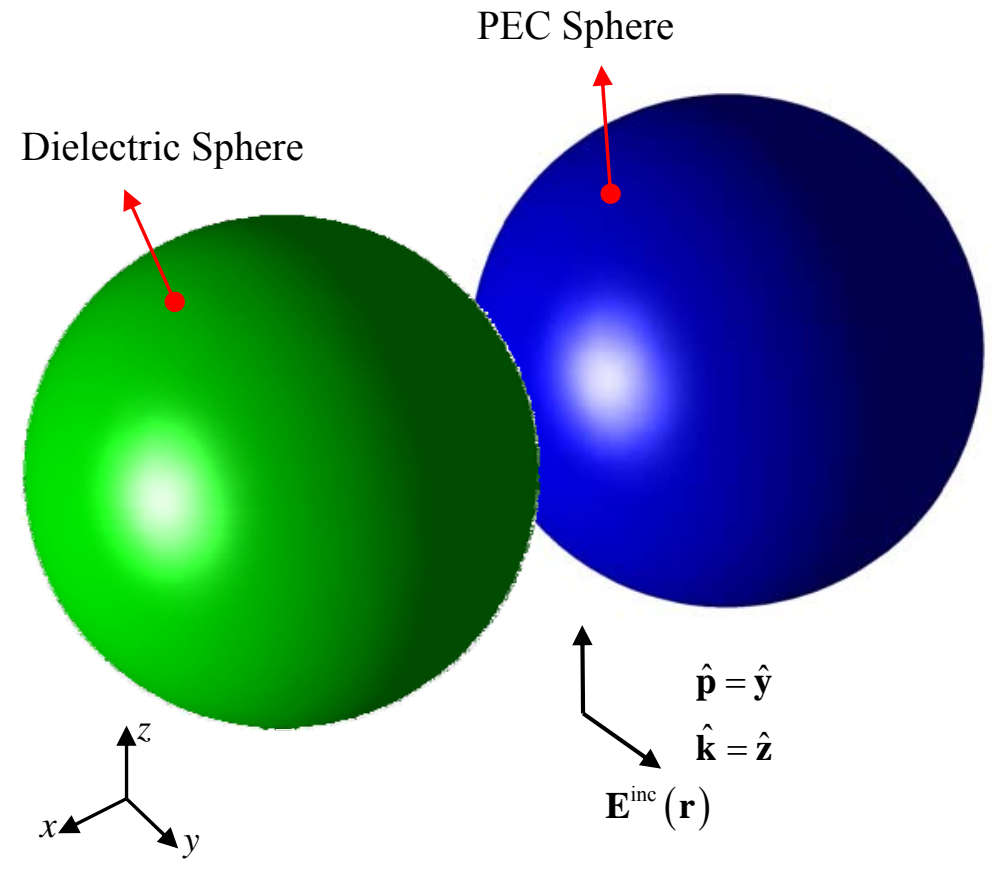

Figure 3(a) 


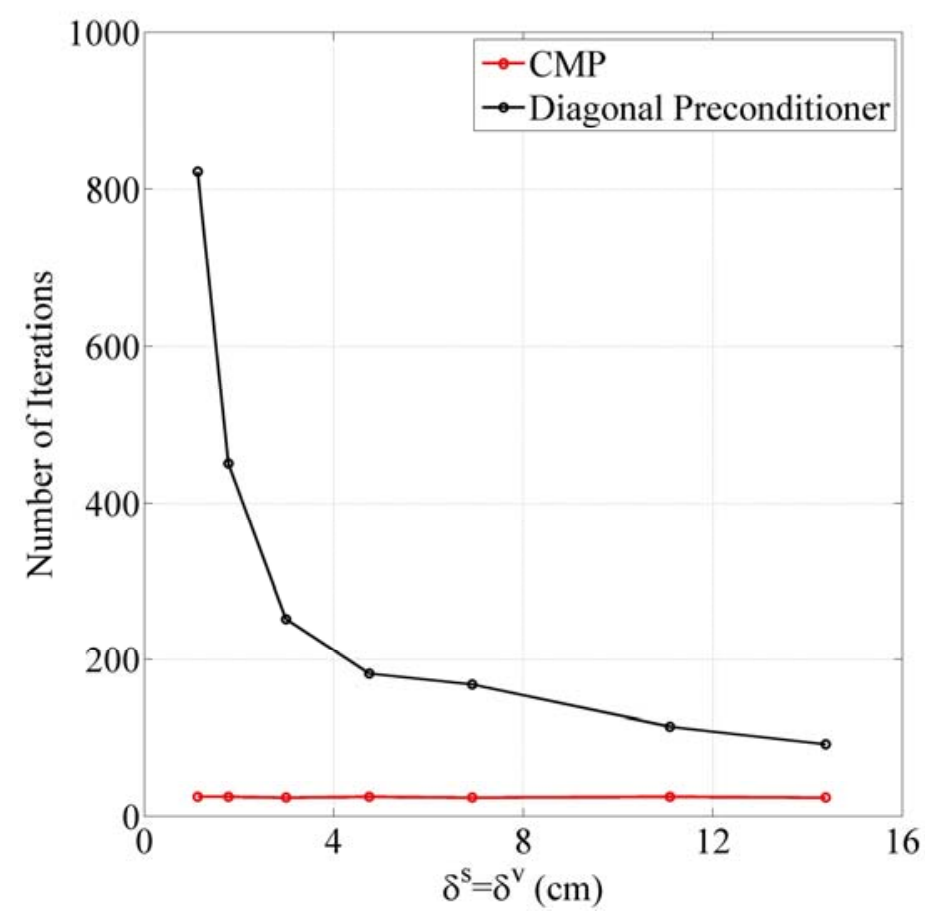

Figure 3(b)

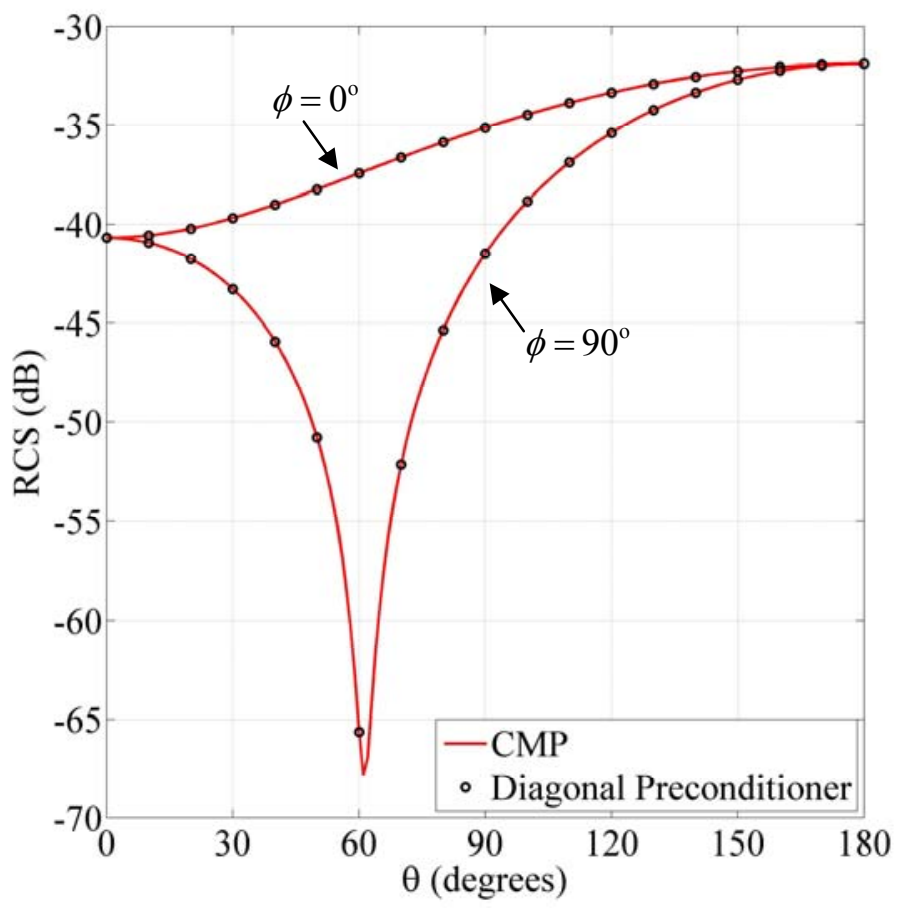

Figure 3(c) 


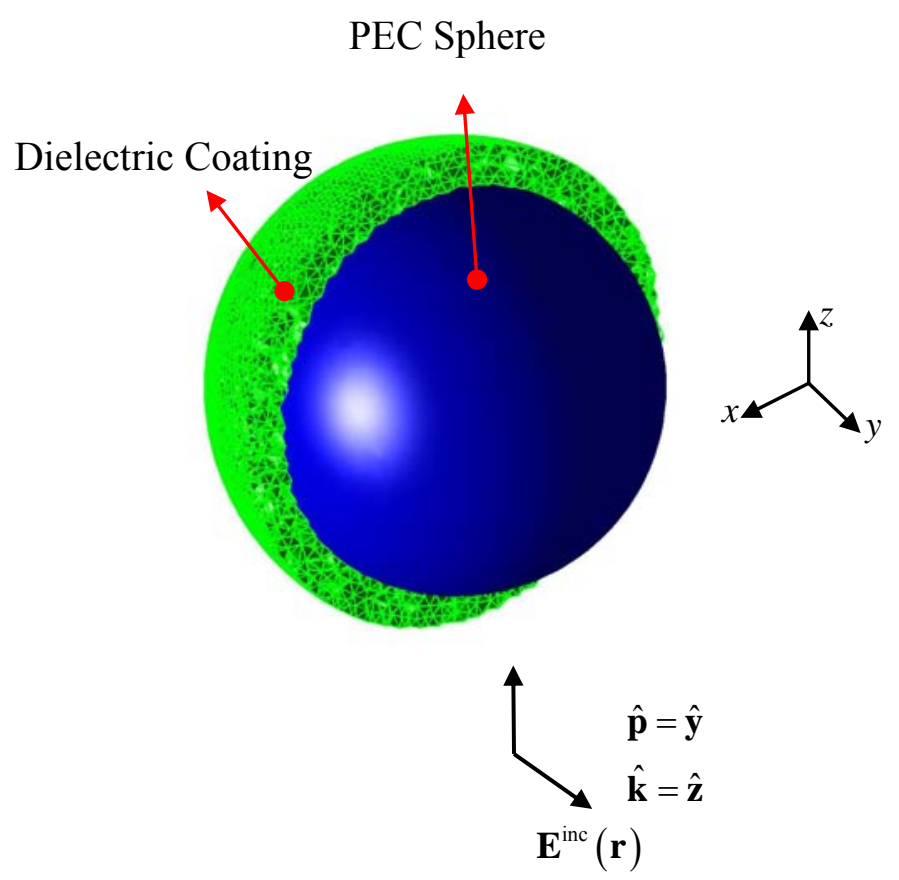

Figure 4(a)

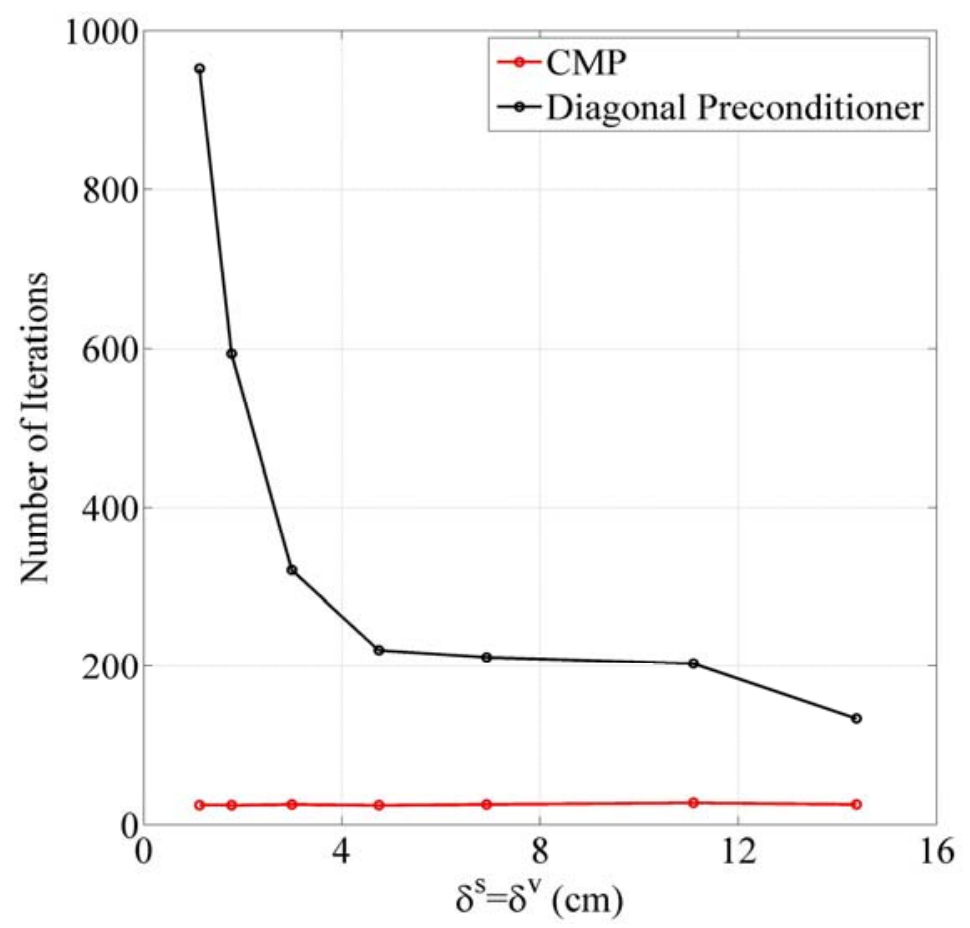

Figure 4(b) 


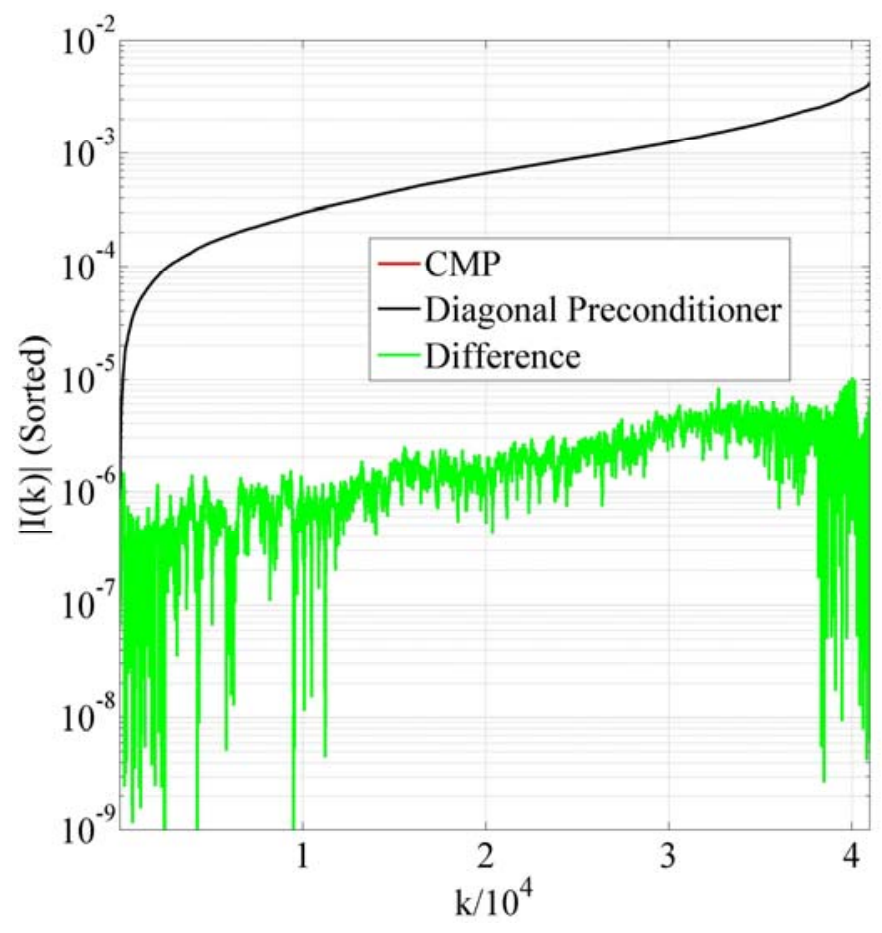

Figure 4(c)

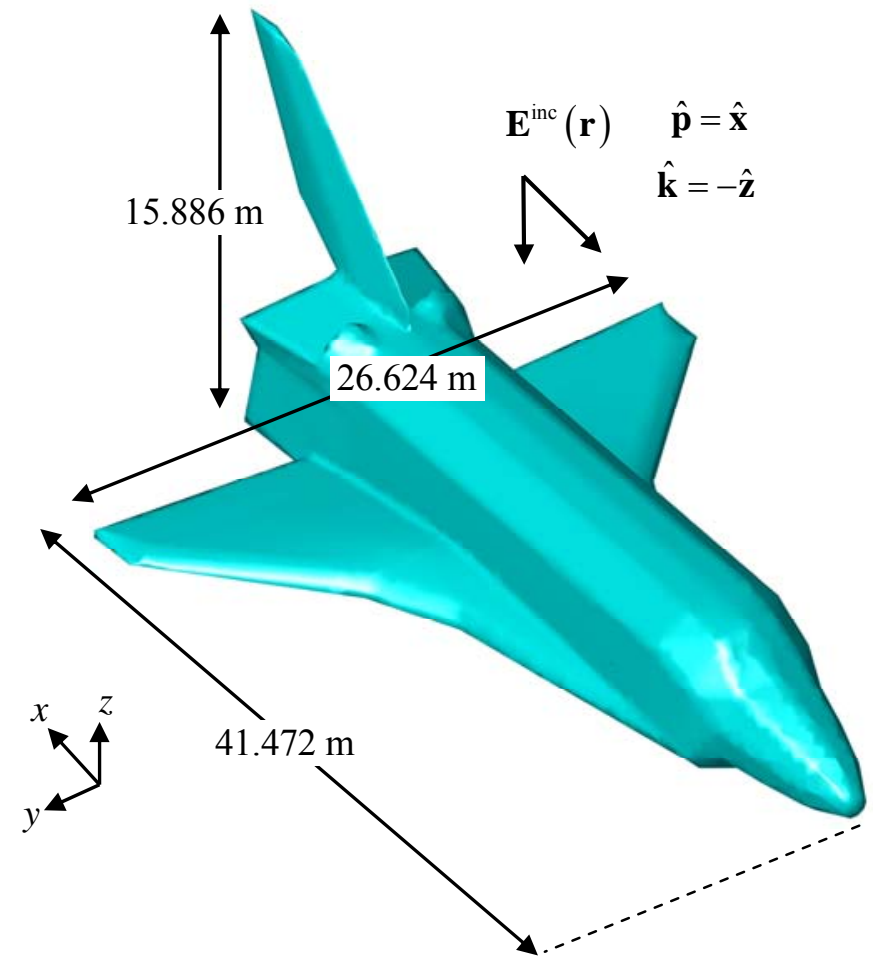

Figure 5(a) 


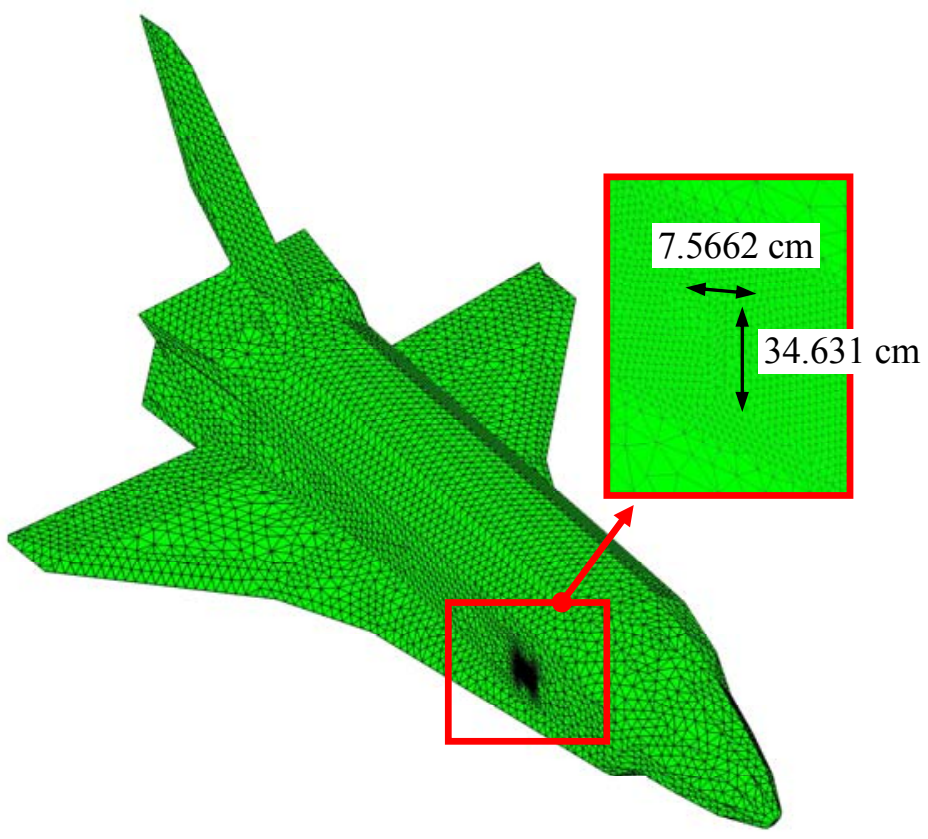

Figure 5(b)

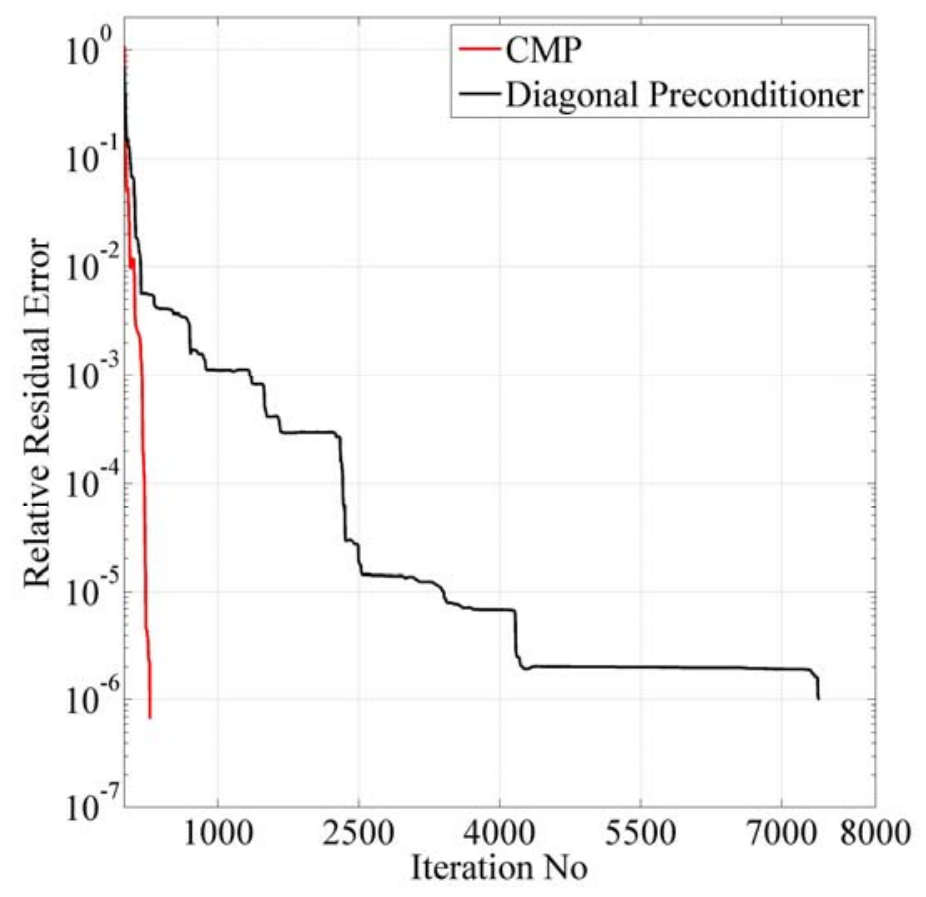

Figure 5(c) 


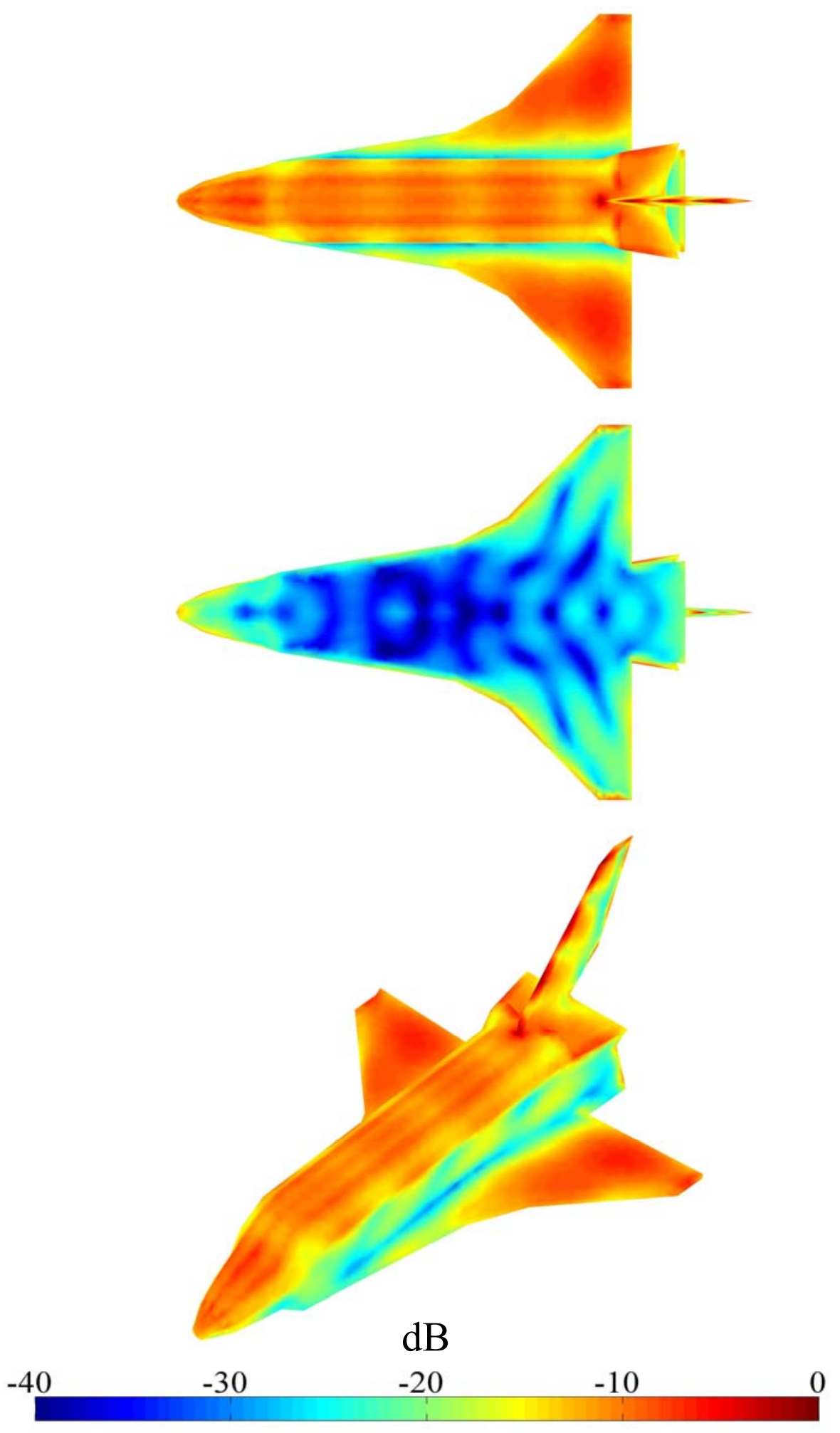

Figure 5(e) 


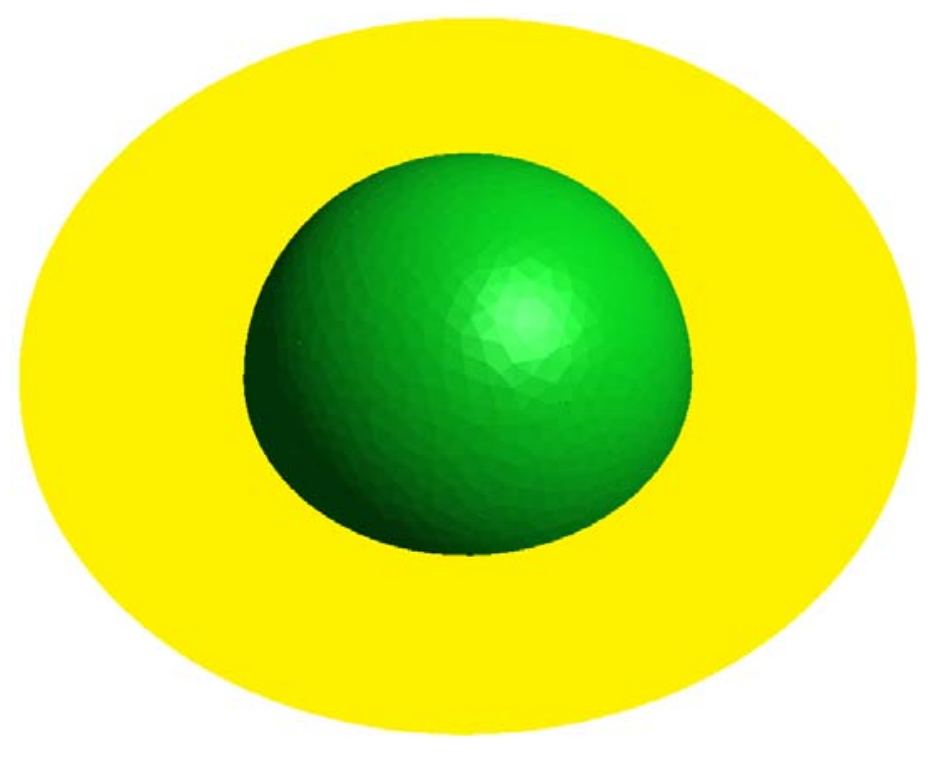

Figure 6 (a)

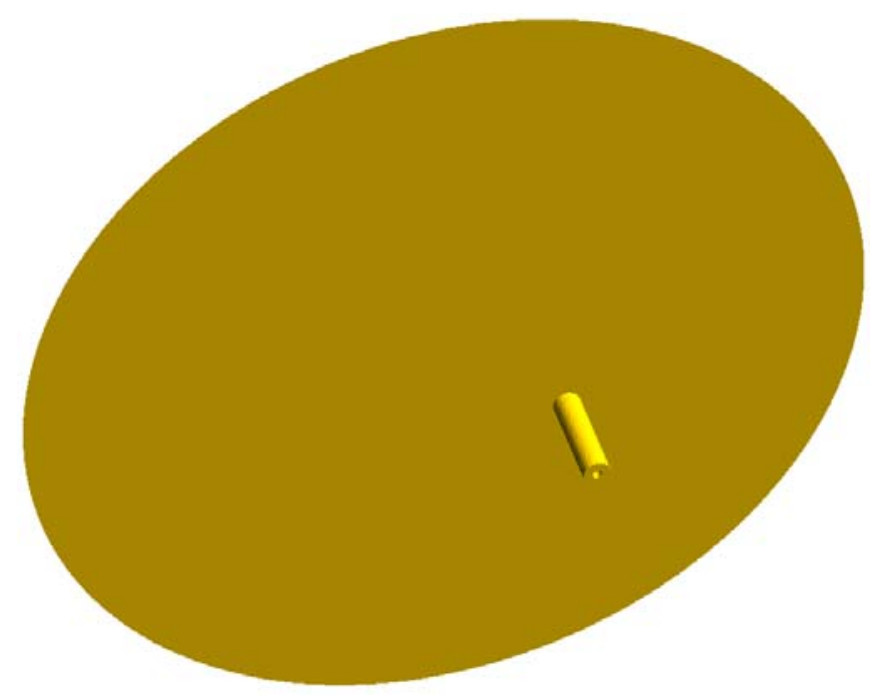

Figure 6 (b) 


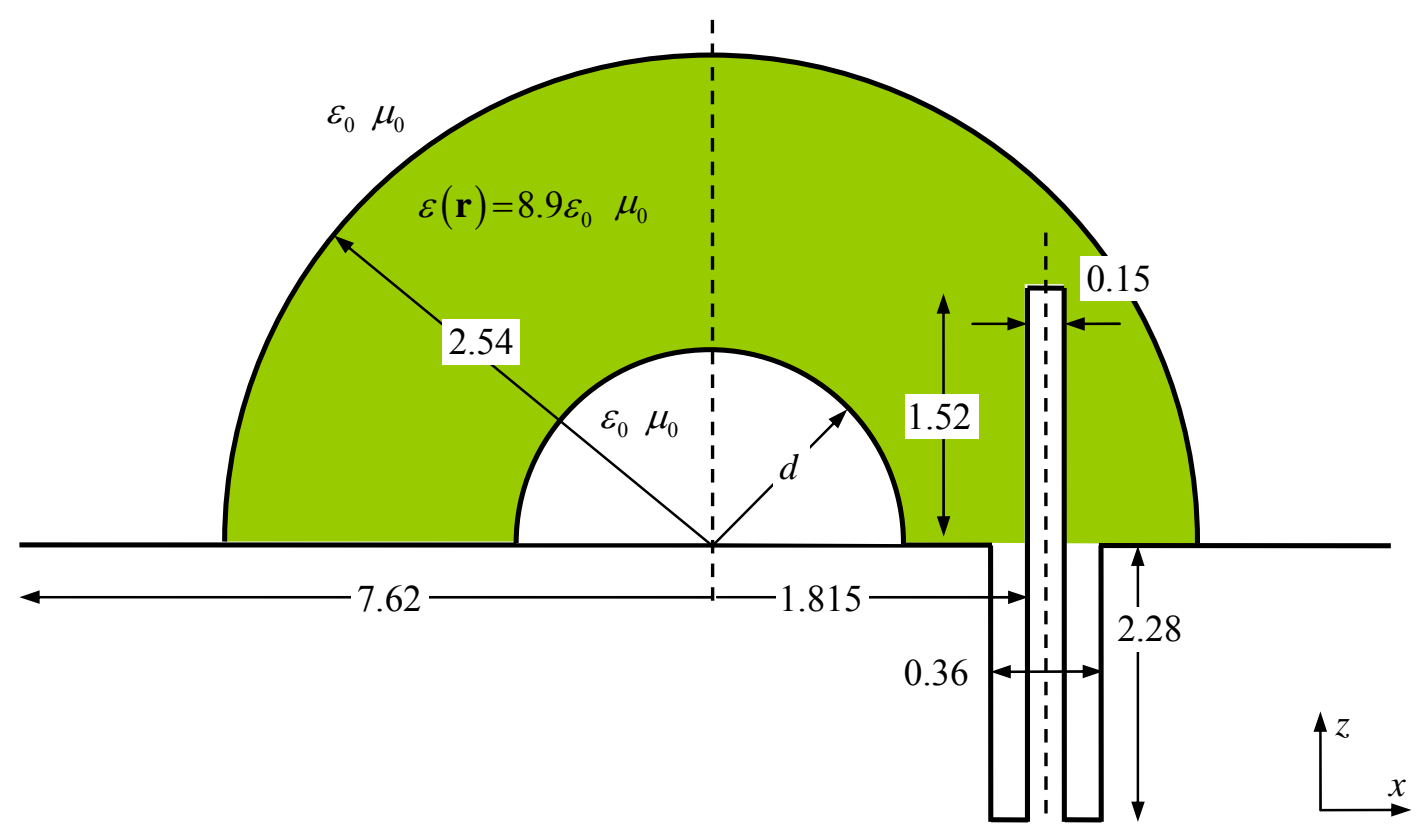

Figure 6 (c)

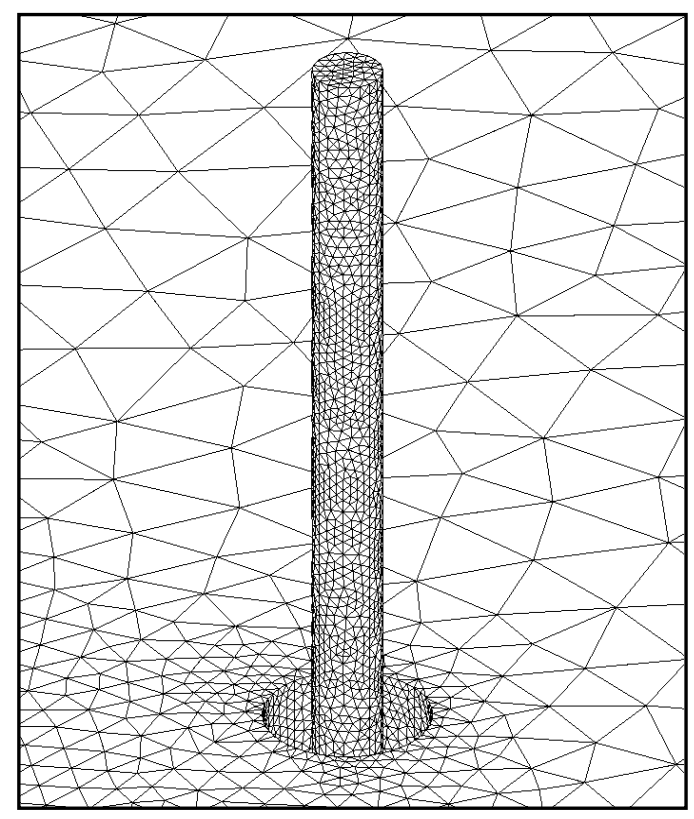

Figure 6 (d) 


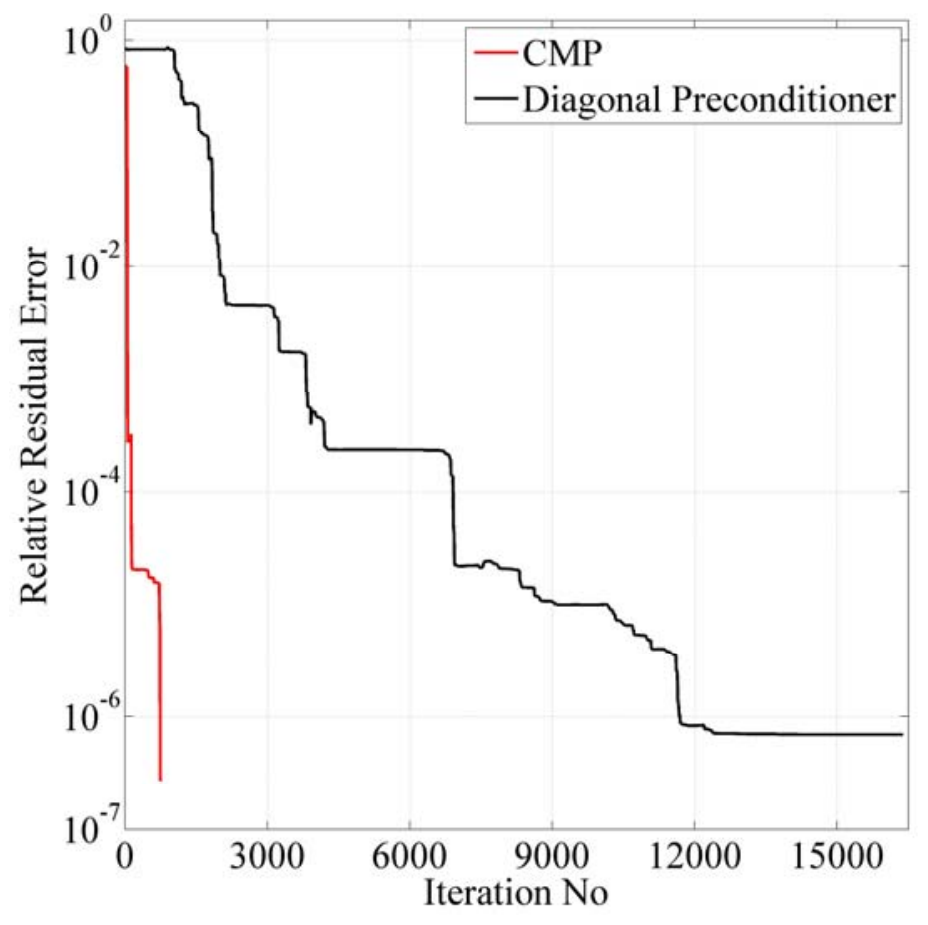

Figure 6 (e)

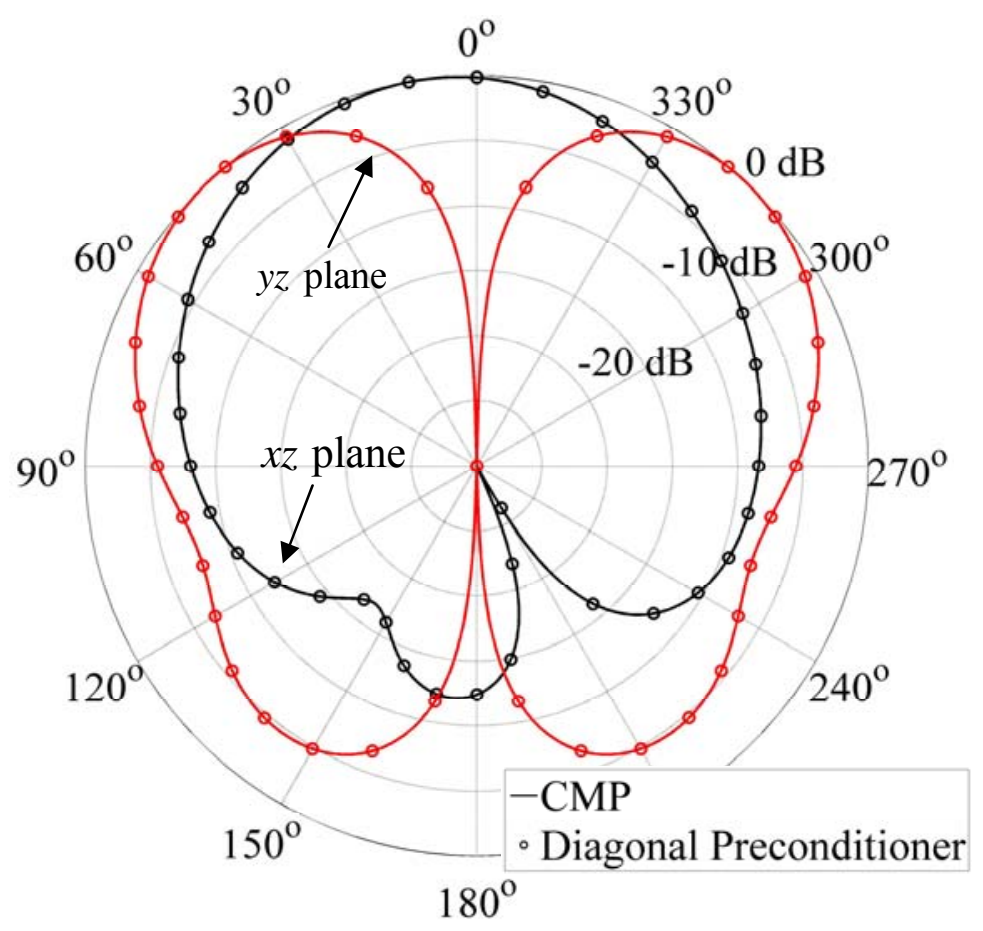

Figure 6 (f) 


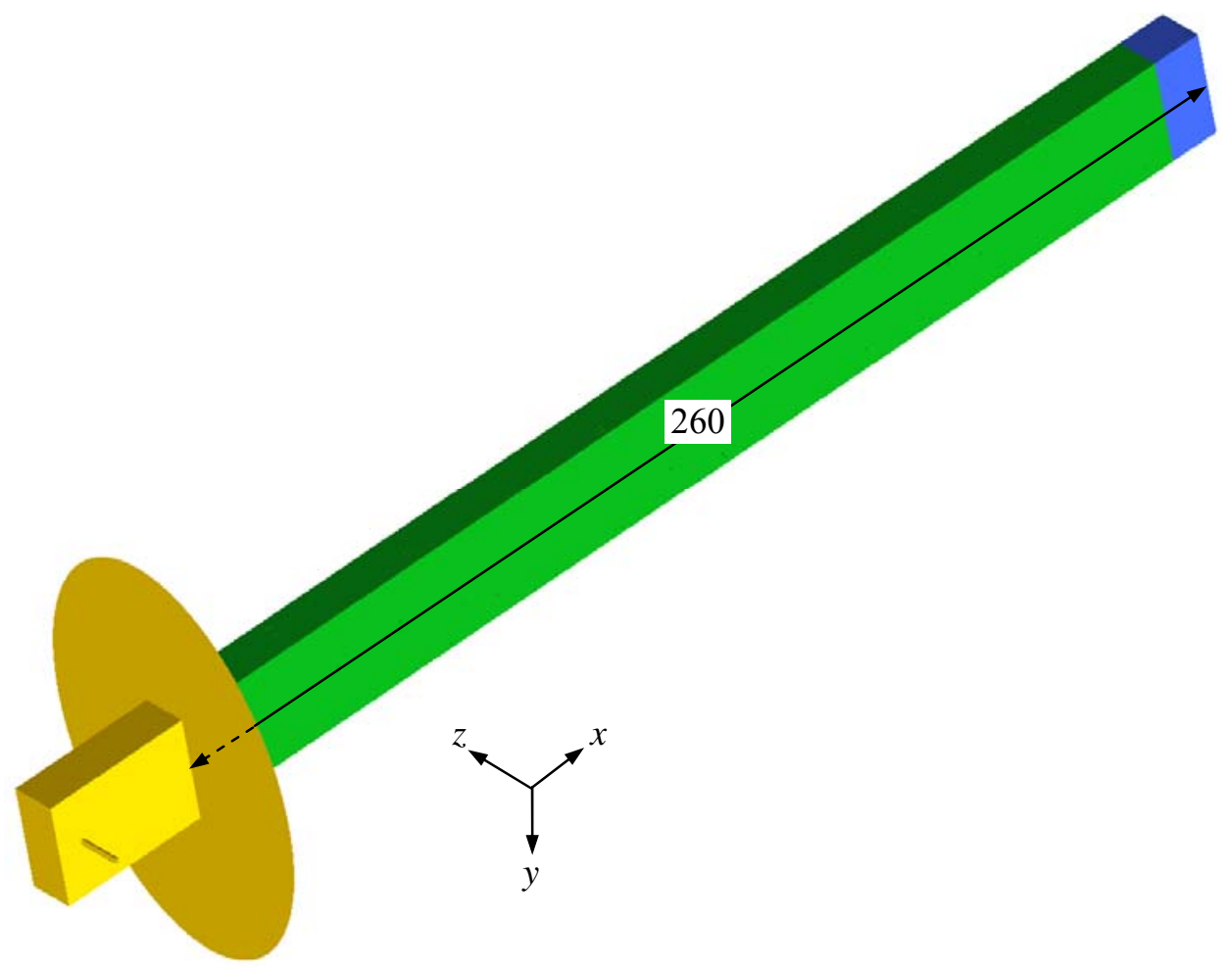

Figure 7 (a)
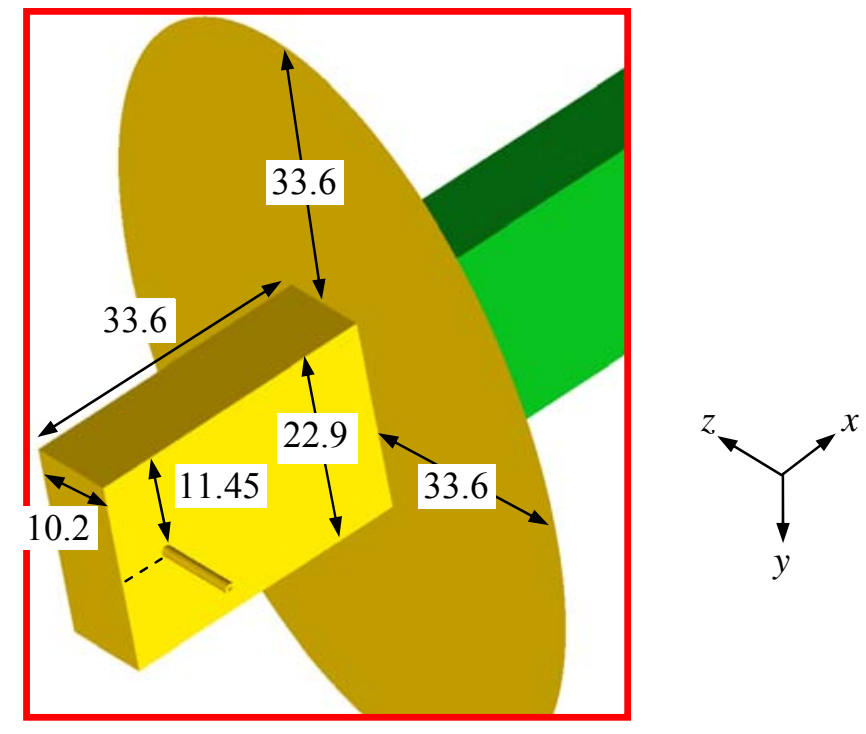

Figure 7 (b) 


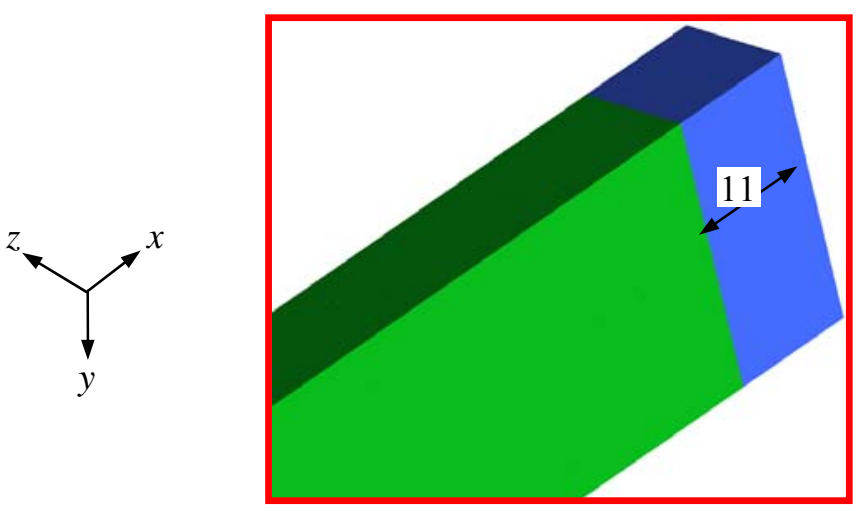

Figure 7 (c)

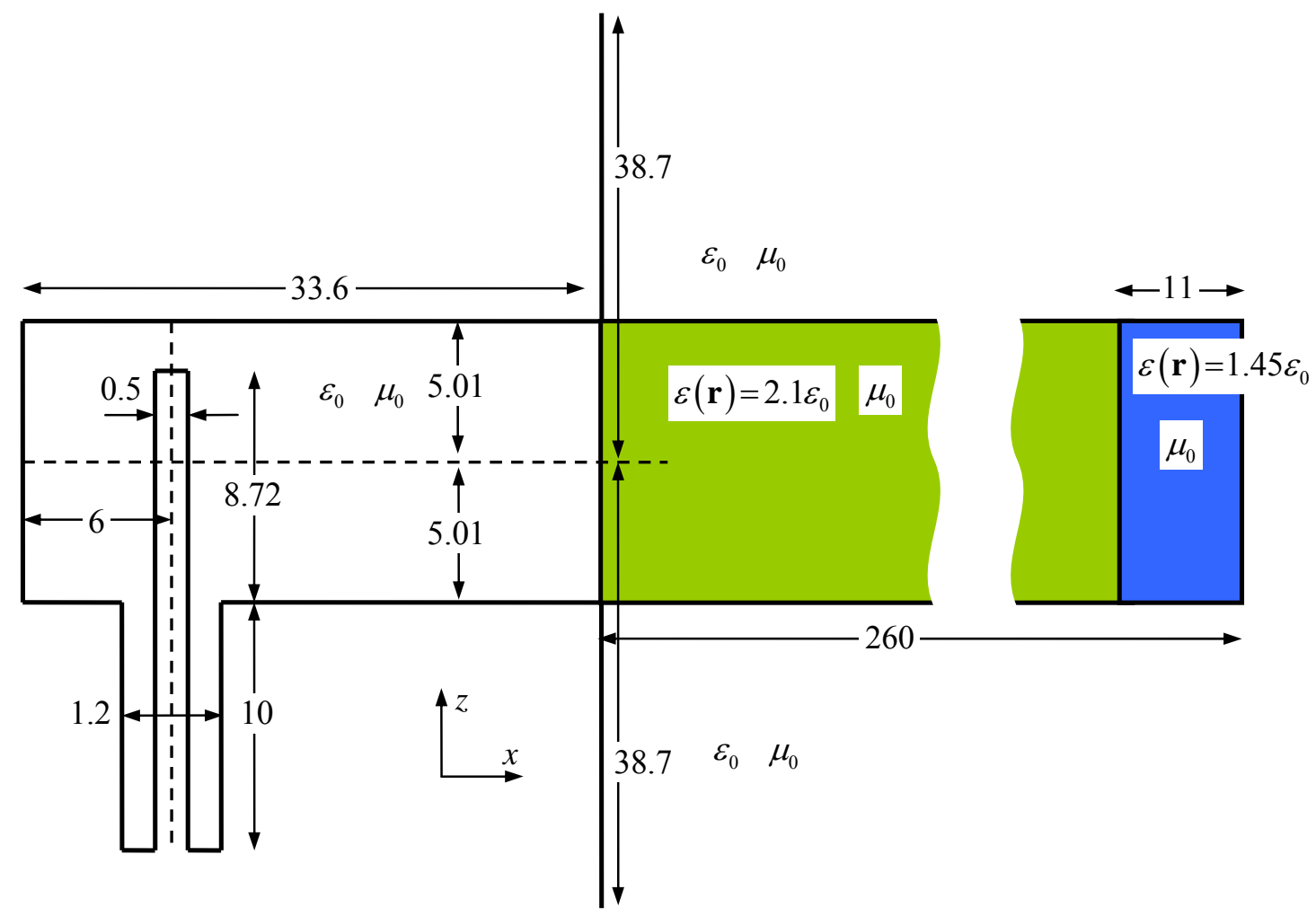

Figure 7 (d) 


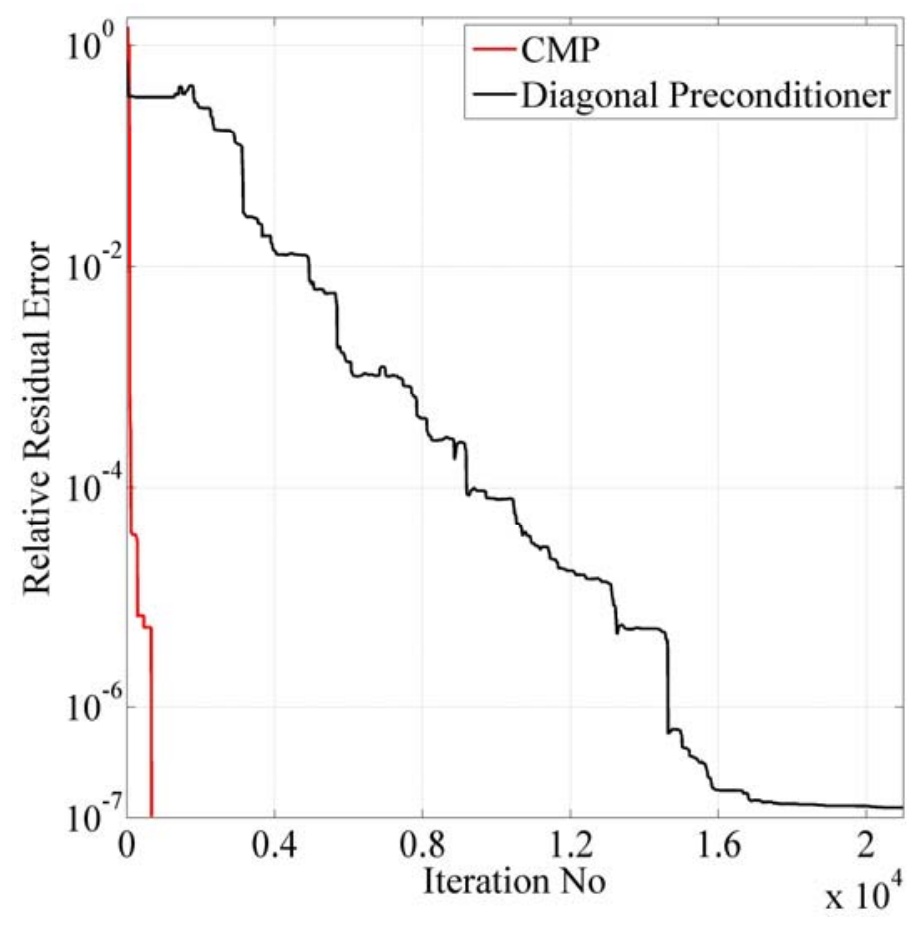

Figure 7 (e)

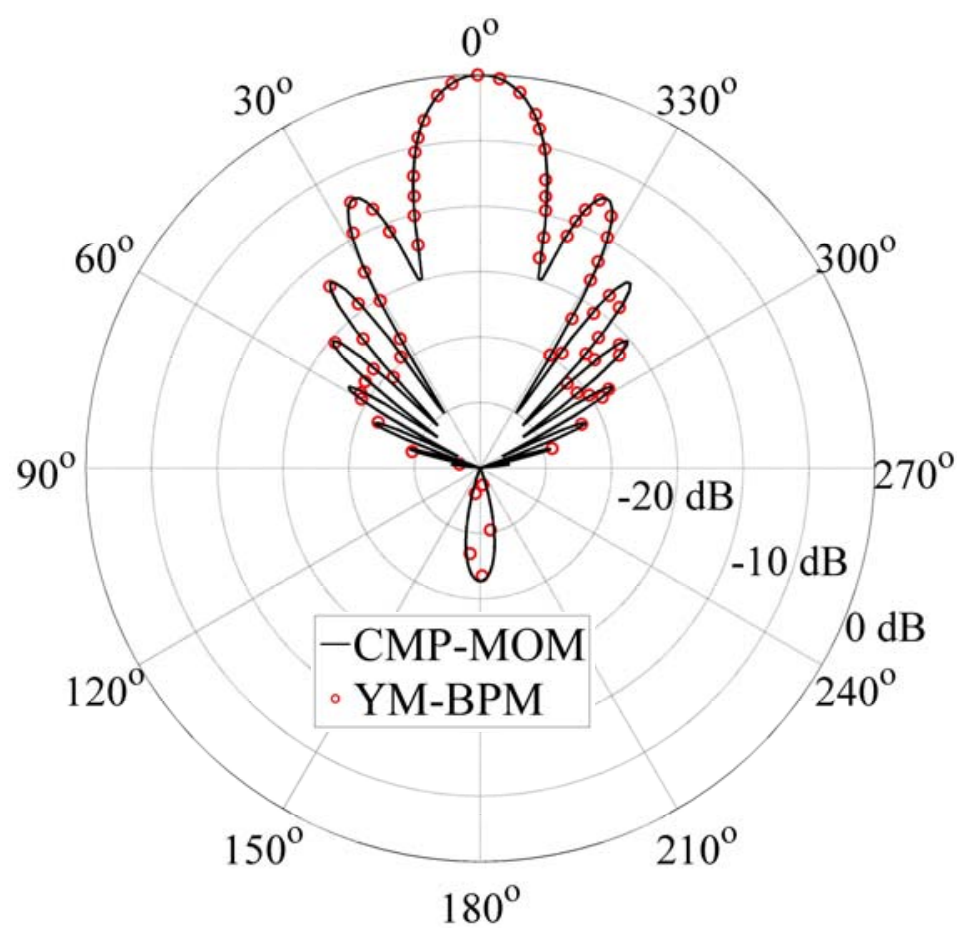

Figure 7 (f) 


\section{References}

[1] C. C. Lu and W. C. Chew, "A coupled surface-volume integral equation approach for the calculation of electromagnetic scattering from composite metallic and material targets," IEEE Trans. Antennas Propagat., vol. 48, no. 12, pp. 1866-1868, Dec. 2000.

[2] A. E. Yilmaz, J.-M. Jin, and E. Michielssen, "A parallel FFT accelerated transient fieldcircuit simulator," IEEE Trans. Microwave Theory Tech., vol. 53, no. 9, pp. 2851-2865, Sept. 2005.

[3] T. K. Sarkar, S. M. Rao, and A. R. Djordjevic, "Electromagnetic scattering and radiation from finite microstrip structures," IEEE Trans. Microwave Theory Tech., vol. 22, pp. 1568-1575, Nov. 1990.

[4] N. V. Budko and A. B. Samokhin, "Spectrum of the volume integral operator of electromagnetic scattering," SIAM J. Sci. Comput., vol. 28, no. 2, pp. 682-700, 2006.

[5] J. Rahola, "On the eigenvalues of the volume integral operator of electromagnetic scattering," SIAM J. Sci. Comput., vol. 21, no. 5, pp. 1740-1754, 2000.

[6] J.-C. Nedelec, Acoustic and Electromagnetic Equations. New York: Springer-Verlag, 2000.

[7] R. J. Adams, "Physical and analytical properties of a stabilized electric field integral equation," IEEE Trans. Antennas Propagat., vol. 52, no. 2, pp. 362-372, Feb. 2004.

[8] F. P. Andriulli, K. Cools, H. Bagci, F. Olyslager, A. Buffa, S. Christiansen, and E. Michielssen, "A multiplicative Calderon preconditioner for the electric field integral equation," IEEE Trans. Antennas Propagat., vol. 56, no. 8, pp. 2398-2412, Aug. 2008.

[9] S. Borel, D. P. Levadoux, and F. Alouges, "A new well-conditioned integral formulation for Maxwell equations in three dimensions," IEEE Trans. Antennas Propagat., vol. 53, no. 9, pp. 2995-3004, Sep. 2005.

[10] H. Contopanagos, B. Dempart, M. Epton, J. Ottusch, V. Rokhlin, J. Visher, and S. M. Wandzura, "Well-conditioned boundary integral equations for three-dimensional electromagnetic scattering," IEEE Trans. Electromagn. Compat., vol. 50, no. 12, pp. 1824-1930, Dec. 2002.

[11] S. M. Rao, D. R. Wilton, and A. W. Glisson, "Electromagnetic scattering by surfaces of arbitrary shape," IEEE Trans. Antennas Propagat., vol. 30, no. 3, pp. 409-418, May 1982.

[12] D. H. Schaubert, D. R. Wilton, and A. W. Glisson, "A tetrahedral modeling method for electromagnetic scattering by arbitrarily shaped inhomogenous dielectric bodies," IEEE Trans. Antennas Propagat., vol. 32, no. 1, pp. 77-85, Jan. 1984.

[13] E. Bleszynski, M. Bleszynski, and T. Jaroszewic, "AIM:Adaptive integral method for solving large-scale electromagnetic scattering and radiation problems," Radio Sci., vol. 31, no. 5, pp. 1225-151, Sept./Oct. 1996.

[14] W. C. Chew, J. M. Jin, C. C. Lu, E. Michielssen, and J. M. Song, "Fast solution methods in electromagnetics," IEEE Trans. Antennas Propagat., vol. 45, no. 3, pp. 533-543, Mar. 1997.

[15] J. M. Song, C.-C. Lu, and W. C. Chew, "Multilevel fast multipole algorithm for electromagnetic scattering by large complex objects," IEEE Trans. Antennas Propagat., vol. 45, no. 10, pp. 1488-1493, Oct. 1997.

[16] G. C. Hsiao and R. E. Kleinman, "Mathematical foundations for error estimation in numerical solutions of integral equations in electromagnetics," IEEE Trans. Antennas Propagat., vol. 45, no. 3, pp. 316-328, Mar. 1997. 
[17] A. Buffa and S. H. Christiansen, "A dual finite element complex on the barycentric refinement," Math. Comput., vol. 76, pp. 1743-1769, 2007.

[18] R. W. Freund, "A transpose-free quasi-minimal residual algorithm for non-hermitian linear systems," SIAM J. Sci. Stat. Comput., vol. 14, pp. 470-482, Mar. 1993.

[19] T. Ando, J. Yamauchi, and H. Nakano, "Numerical analysis of a dielectric rod antenna Demonstration of the discontinuity-radiation concept," IEEE Trans. Antennas Propagat., vol. 51, no. 8, pp. 2007-2013, Aug. 20032003. 